\title{
Complement C1s and C4d as prognostic biomarkers in renal cancer: emergence of non- canonical functions of $\mathrm{C} 1 \mathrm{~s}$
}

Marie V. Daugan ${ }^{1, \#}$, Margot Revel ${ }^{1, \#}$, Jules Russick ${ }^{1}$, Marie-Agnes Dragon-Durey ${ }^{1,2,3}$, Christine Gaboriaud $^{4}$, Tania Robe-Rybkine ${ }^{1}$, Victoria Poillerat ${ }^{1}$, Anne Grunenwald ${ }^{1}$, Guillaume Lacroix ${ }^{1}$, Antoine Bougouin ${ }^{1}$, Maxime Meylan ${ }^{1}$, Virginie Verkarre ${ }^{2,6}$, Stephane M. Oudard ${ }^{7}$, Arnaud Mejean ${ }^{2,7}$, Yann A. Vano $^{1,2,6}$, Geraldine Perkins ${ }^{8}$, Pierre Validire ${ }^{9}$, Xavier Cathelineau ${ }^{2,10}$, Rafael Sanchez-Salas ${ }^{10}$, Diane Damotte $^{1,2,11}$, Veronique Fremeaux-Bacchi ${ }^{1,3}$, Isabelle Cremer $^{1}$, Catherine Sautès-Fridman ${ }^{1}$, Wolf H. Fridman $^{1}$, Lubka T. Roumenina ${ }^{1} *$

${ }^{1}$ Centre de Recherche des Cordeliers, INSERM, Sorbonne Université, Université de Paris, F-75006 Paris, France.

${ }^{2}$ Université de Paris, F-75006 Paris, France.

${ }^{3}$ Hôpital Européen Georges-Pompidou, Biological Immunology Department, Assistance Publique Hopitaux de Paris, France.

${ }^{4}$ University Grenoble Alpes, CNRS, CEA, IBS, F-38000 Grenoble, France

${ }^{5}$ Hôpital Européen Georges-Pompidou, Pathology Department, Assistance Publique Hopitaux de Paris, F-75015, France.

${ }^{6}$ Hôpital Européen Georges-Pompidou, Oncology Department, Assistance Publique Hopitaux de Paris, France.

${ }^{7}$ Hôpital Européen Georges-Pompidou, Urology Department, Assistance Publique Hopitaux de Paris, F-75015, France.

${ }^{8}$ Hôpital Européen Georges-Pompidou, Gastroenterology and Hepatology Department, Assistance Publique Hopitaux de Paris, F-75015, France.

${ }^{9}$ Institut Mutualiste Montsouris, Department of Pathology, Paris, France.

${ }^{10}$ Institut Mutualiste Montsouris, Department of Urology, Paris, France.

${ }^{11}$ Hôpital Cochin, Departments of Pathology and Thoracic Surgery, Assistance Publique Hopitaux de Paris, F-75014 France.

\# These authors contributed equally to this study.

"Correspondence to: Lubka T. Roumenina, Ph.D.; Cordeliers Research Center, INSERM UMRS 1138; 15 rue de l'Ecole de Medecine; 75006 Paris, France. Phone: 33-1-44-27-90-96/ Fax: 33-1-40-51-04-20, Email: lubka.roumenina@sorbonne-universite.fr 
Disclosures: The authors declare no potential conflicts of interest.

Funding Information: This work was supported by grants from the Ligue Regionale Contre le Cancer and Fondation ARC Pour La Recherche Sur Le Cancer to LTR. This work was also supported also by grants from CARPEM (ExhauCRF program), INCa (HTE program), Canceropole lle de France (R17054DD), Association pour la recherche en thérapeutiques innovantes en cancérologie (ARTIC) to CSF, French National Research Agency grant (ANR-16-CE91-0004-01) to CG. The Labex ImmunoOncology Excellence Program, INSERM, University of Paris and Sorbonne University also supported this work. MVD received a PhD fellowship from La Fondation ARC pour la recherche sur le cancer. AG received a PhD fellowship from Fondation pour la Recherche Médicale - FRM.

Key words: complement system, clear cell renal cell cancer, C1s, plasma C4d, non-canonical functions of complement

Synopsis: This clinical study shows C1s drives renal cell-carcinoma progression by local complement activation and complement cascade-unrelated, intracellular, non-canonical functions. This modulates the tumor microenvironment by impacting tumor cell proliferation, survival and interaction with T cells. 


\section{Abstract}

The complement system plays a complex role in cancer. In clear cell renal cell carcinoma (ccRCC), local production of complement proteins drives tumor progression, but the mechanisms by which they do this are poorly understood. We found that complement activation, as reflected by high plasma C4d or as C4d deposits at the tumor site, was associated with poor prognosis in 2 cohorts of patients with ccRCC. High expression of the C4-activating enzyme C1s by tumor cells was associated with poor prognosis in 3 cohorts. Multivariate Cox analysis revealed that the prognostic value of $\mathrm{C} 1 \mathrm{~s}$ was independent from complement deposits, suggesting the possibility of complement cascadeunrelated, pro-tumoral functions for $\mathrm{C} 1 \mathrm{~s}$. Silencing of $\mathrm{C} 1 \mathrm{~s}$ in cancer cell lines resulted in decreased proliferation and viability of the cells and in increased activation of T cells in in vitro co-cultures. Tumors expressing high levels of C1s showed high infiltration of macrophages and $\mathrm{T}$ cells. Modification of the tumor cell phenotype and T-cell activation were independent of extracellular C1s levels, suggesting that $\mathrm{C} 1 \mathrm{~s}$ was acting in an intracellular, non-canonical manner. In conclusion, our data point to $\mathrm{C} 1 \mathrm{~s}$ playing a dual role in promoting ccRCC progression by triggering complement activation and by modulating the tumor cell phenotype and tumor microenvironment in a complement cascade-independent, non-canonical manner. Overexpression of $\mathrm{C} 1 \mathrm{~s}$ by tumor cells could be a new escape mechanism to promote tumor progression. 


\section{Introduction}

The complement system is a first line of immune defence against pathogens [1]. In cancer, the complement system plays a context-dependent role, either killing tumor cells or generating local chronic inflammation, thereby promoting tumor growth [2]. Analysis of the prognostic impact of intratumoral expression of complement genes has revealed the existence of tumors with "protective" or "aggressive" complement signatures, illustrating the context-dependent role of the complement system in cancer. Key genes overexpressed in the "aggressive" signature are components of the classical complement pathway encoding $\mathrm{C} 1 \mathrm{q}, \mathrm{C} 1 \mathrm{r}, \mathrm{C} 1 \mathrm{~s}, \mathrm{C} 4$ and $\mathrm{C} 3$. $\mathrm{C} 1 \mathrm{q}$ interacts with $\mathrm{C} 1 \mathrm{r}$ and $\mathrm{C} 1 \mathrm{~s}$ to form the complement-initiating $\mathrm{C} 1$ complex [3]. Upon recognition of immune complexes by C1q, C1s becomes activated and cleaves the components $\mathrm{C} 4$ and $\mathrm{C} 2$ to initiate the cascade [4-7]. In clear cell renal cell carcinoma (ccRCC), tumor cells that produce C1s and C1r can hijack C1q generated by macrophages to initiate the classical complement pathway locally on IgG, deposited on the tumor cell surface [8]. Classical pathway activation, as measured by deposits of C4 activation fragments in tumor sections, is associated with a poor prognosis in non-small cell lung cancer (NSCLC) [9] and cCRCC [8], suggesting a canonical complement cascade-related mechanism. Moreover, the level of $\mathrm{C} 4 \mathrm{~d}$, a cleavage product of $\mathrm{C4}$, is elevated in the plasma of patients with NSCLC $[9,10]$ and malignant pleural mesothelioma [11] and can predict patient survival. Overexpression of C1s also independently predicts poor disease-specific and metastasis-free survival rates in urothelial carcinoma [12], although the mechanism responsible remains unknown.

Mouse fibroblasts, which do not produce $\mathrm{C} 1 \mathrm{~s}$ and do not form tumors when injected in vivo, gain tumorigenic capacity when transfected with the C1s gene [13]. This tumorigenic potential is dependent on the enzymatic activity of C1s [14]. C1s knockdown in cutaneous squamous cell carcinoma (CSCC) [15] and glioblastoma [16] cell lines promotes apoptosis and significantly suppresses growth of human CSCC xenograft tumors in vivo. In contrast, C1s overexpression correlates with decreased survival in glioblastoma [16]. Based on these results, we hypothesized the existence of pro-tumoral, cascade-independent functions of $\mathrm{C} 1 \mathrm{~s}$, which could be considered as noncanonical. However, the extent to which the canonical and non-canonical functions of complement proteins contribute to tumor progression is unknown.

Here, we have shown that a fraction of cancer cells in ccRCC tumors exhibited a complement-rich phenotype, including high levels of the initiating enzyme C1s. C1s overexpression by tumor cells was associated with shorter survival. The pro-tumoral effect of $\mathrm{C} 1 \mathrm{~s}$ was found to involve three major mechanisms: 1) a canonical function of cleaving $\mathrm{C} 4$ and initiating the classical pathway; 2) a function that includes non-canonical, intracellular activity that directly affects the tumor cell transcriptome, phenotype and capacity to 3 ) activate T cells. Thus, C1s can promote tumor cell proliferation and escape from immunosurveillance through modulation of the tumor microenvironment. 


\section{Material and methods}

\section{Patients}

The patients included in this study signed an informed consent form prior to inclusion. The research was approved ( $\mathrm{N}^{\circ}$ CEPAR 2014-001, CPP Ile-de-France II $\mathrm{n}^{\circ} 2016-07-08$ ) by the medical ethics board and conducted according to the recommendations in the Helsinki Declaration. Three independent cohorts of primary ccRCC patients undergoing nephrectomy were evaluated. The first cohort (cohort 1) was a retrospective cohort of 82 patients who underwent surgery at Necker-Enfants Malades Hospital (Paris, France) between 1999 and 2003 [17]. The second cohort (cohort 2) was a prospective cohort of 26 patients for whom plasma-EDTA samples were collected at time of surgery when the tumor size was greater than $4 \mathrm{~cm}$ at Hospital Européen Georges Pompidou (Paris, France) since 2017. The third cohort (cohort 3) included 92 patients since 2014 whose tumors were collected at the time of surgery at Institut Mutualiste Montsouris (IMM, Paris, France). Among these patients, we assessed 58 plasma-EDTA samples. Histologic subtype of ccRCC, all TNM stages and tissue with good quality for the analysis constituted the inclusion criteria. Histopathologic features such as Fuhrman grade, TNM stages and size were available for the majority of the patients (Supplementary table S1). As controls for C4d quantification by ELISA, 31 plasma-EDTA samples from healthy donors were used for cohort 3 and six for cohort 2. Sections from one NSCLC tumor were available from the Departments of Pathology and Thoracic Surgery, Cochin Hospital, Paris.

\section{C4d quantification in plasma}

A commercial kit was used to measure C4d in plasma (Svar, cat\# COMPL C4d RUO). Plasma-EDTA samples were stored at $-80^{\circ} \mathrm{C}$ until use. The quantification was done according to manufacturer's instructions using the reagents of the kit. In brief, plasma samples were diluted 1:50 and transferred to microtiter wells pre-coated with the C4d-specific monoclonal antibody for 1 hour. After washing, incubation with the secondary antibody labelled with HRP was performed for 30 minutes. After washing, the substrate was added for 30 minutes, and the colour was measured usinf an ELISA plate reader Infinite 200 PRO (Tecan). Concentrations were calculated as described by the manufacturer of COMPL C4d RUO, taking into account the 1:50 dilution.

\section{ELISA for C1s quantification}

An ELISA assay for quantification of C1s was set up in house using C1s-specific IgG, purified using Protein G resin (GE Healthcare, cat\# 17061802) from polyclonal goat anti-human C1s antiserum (Quidel, cat\# A302), which were left native or were biotinylated with EZ link NHS Biotin according to the manufacturer's instructions (Thermo Fisher, cat\# 20217). Briefly, Maxisorb ELISA plates were coated with native anti-C1s IgG for $2 \mathrm{~h}$ at room temperature and then blocked with PBS 5\% BSA. Pure supernatants and cell lysates were added and incubated for $1 \mathrm{~h}$ at room temperature. After washing 
with PBS-0.05\% Tween 20, biotinylated anti-C1s IgG was applied for $1 \mathrm{~h}$ at room temperature followed by Strepavidin-HRP (Dako, cat\# P0397). Binding was detected with TMB Microwell Peroxidase (Sera Care, cat\# 5120-0075) and stopped after 10min at room temperature with $1 \mathrm{M}$ sulphuric acid. The absorbance was read using a Tecan infinite M200 Pro. Purified C1s (Complement Technology, cat\# A103) was used as a standard.

\section{Immunofluorescence (IF)/immunohistochemistry (IHC)}

Formalin-fixed paraffin-embedded (FFPE) human cCRCC or NSCLC tumors were cut into 3- $\mu$ m-thick sections and stained for C1s, C1q, C4, C3, C5aR1, Cytokeratin, Vimentin, MHC class I, aSMA, CD31, CD68, CD163, CD66b, CD20, CD3, or CD8. The primary and secondary antibodies used, as well as the antigen retrieval and staining conditions, are summarized in Supplementary Table S2. Human liver sections (Geneticist) were used as a positive control for $\mathrm{C} 1 \mathrm{~s}, \mathrm{C} 4$ and $\mathrm{C} 3$. Tonsil (Geneticist) was used as a positive control for C1q, cytokeratin, aSMA, CD31, CD163, CD20, and CD3. First, tissues were deparaffinized with three baths of Clearene (Leica Biosystem, cat\# 3803600E) and rehydrated with an ethanol gradient (100\%, 90\%, 70\%, 50\%). Second, PT-link (Dako, PT200) was used for antigen retrieval with heat for 30 minutes at $97^{\circ} \mathrm{C}$ in EnVision FLEX Retrieval Solution (Dako, pH Low cat\# K8005, pH High cat\# K8004) at high or low pH depending of the antibody (Supplementary Table S2). A $3 \% \mathrm{H}_{2} \mathrm{O}_{2}$ solution (Gilbert, cat\# 6553036) was added for blocking of endogenous peroxidase, and nonspecific binding was inhibited by protein blocking (Dako, cat\# X0909). For IHC, 3-amino-9ethylcarbazole substrate (Vector Laboratories, cat\# SK4200) was used. Slides were then counterstained with haematoxylin (Dako, cat\# CS700), mounted with glycergel (Dako, cat\# C0563) and scanned with a Nanozoomer (Hamamastu). For IF, the nucleus was stained with DAPI; slides were mounted with Prolong Glass antifade reagent and scanned with AxioScan (Zeiss). Calopix software was used to quantify PD1, CD8, and CD163. In all cases, isotype controls were used. The whole slide was always analyzed, avoiding the bias of choosing particular areas.

Staining specificity. A competition test on liver sections was performed to verify the specificity of $\mathrm{C} 1 \mathrm{~s}$ staining (Supplementary Figure S1A-B). In this test, pre-incubation at different molar ratios of anti$\mathrm{C} 1 \mathrm{~s}$ (Abcam, cat\# ab134943) and purified C1s protein for 1 hour at $37^{\circ} \mathrm{C}$ was performed before addition to tissues. Purified C1r (Complement Technology, cat\# A101) served as a negative control.

Patient stratification. Among the patients in cohort 1, 38 were analysed for C1s and 77 for C4 considering sample availability and staining specificity. The tumors were classified into 3 groups in a semi-quantitative manner according to the percentage of C1s-positive tumor cells; $0(<5 \%), 1(5-$ $30 \%), 2(>30 \%)$. For the classification, only the staining on tumor cells was considered. To avoid bias, the stratification was realized by three independent observers (MVD, MR, GL or $A B$ ). As a result of their similar effect on survival, groups 0 and 1 were combined to form a "Low" group and compared to the "High" group, with a score of 2 . The same method was applied for the classification of anti-C4d and anti-C3d staining, as previously described by Roumenina et al. [8]. Briefly, the staining with anti- 
C3d and anti-C4d showed two patterns: membranous staining, identified as deposits of complementactivation fragments, and intracellular staining. The two patterns were distinguished visually by the observers and quantified separately. Independent scores for the "deposits" and for the "intracellular" staining were calculated.

\section{Cell lines}

Human lung adenocarcinoma cells (A549), human ccRCC cells (Caki-1 and A498) and human colon carcinoma cells (HCT116) were purchased from ATCC in 2016 and cultured in a humidified atmosphere of $5 \% \mathrm{CO} 2$ at $37^{\circ} \mathrm{C}$ until $90 \%$ confluence. HCT116 and Caki-1 cells were cultured in McCoy's medium (Gibco, cat\# 16600082) + 10\% heat-inactivated FCS (Biowest, cat\# S1810-500) + 1X penicillin/streptomycin (Gibco, cat\# 10378016). A498 cells were cultured in Eagle minimum essential medium (EMEM) (ATCC, cat \# 30-2003) $+10 \%$ heat-inactivated FCS $+1 X$ penicillin/streptomycin. A549 cells were cultured in Dulbecco's modified eagle medium nutrient mixture F12 (Gibco, cat\# 11320033) $+10 \%$ heat-inactivated FCS +1X penicillin/streptomycin + 1X L-glutamine (Gibco, cat\# 25030081) + 1X HEPES (Gibco, cat\# 15630080) + 1X non-essential amino-acids solution (Gibco, cat\# 11140050). All cell lines were routinely tested for Mycoplasma with a kit (Ozyme, cat\# LT07-710) and used only if negative. The cells were authenticated by morphology before each experiment.

For some experiments, Caki-1 cell lines were cultured for $48 \mathrm{~h}$ with or without IFN $\gamma$ (PreproTech, cat\# $300-02)$ at different concentration (10ng/ml or $100 \mathrm{ng} / \mathrm{ml})$.

\section{Silencing of C1s}

A 30-minute pre-incubation of 4 siRNAs against C1s (Qiagen Hs_C1S_1_Flexitube siRNA cat\# SI00027356; Qiagen Hs_C1S_2_Flexitube siRNA cat\# S100027363; Qiagen Hs_C1S_3_Flexitube siRNA cat\# SI00027370; and Hs_C1s_5_Flexitube siRNA cat\# SI030322873) or siRNA control (Qiagen AllStars negative control siRNA, cat\# SI03650318) at $50 \mathrm{nM}$ with lipofectamine RNAimax (ThermoFisher, cat\# 13778030) in OptiMEM (Gibco, cat\# 31985070) was performed before adding this mixture to cells at $50 \%$ confluence in 6 -well plates. The transfection was stopped after $24 \mathrm{~h}$ by replacing the medium containing siRNA with the standard medium in which the cells were routinely cultured (see Cell lines) without antibiotics. Photographs and functional assays were performed on the silenced cells 72 hours post-transfection. The cells and supernatants were collected to assess the efficacy of silencing by qRT-PCR and western blotting. Cells treated with siRNA against $C 1 S$ were designated siC1s and the cells treated with a control siRNA against an irrelevant sequence were designated siC.

\section{Recombinant C1s-expressing stable cell line HCT116}

Bacteria transformation and plasmid amplification: 
The pcDNA3.1/Zeo C1s plasmid was obtained by insertion of C1s PCR amplicon of pFastBac1/C1s in pcDNA3.1/Zeo between Nhel and EcoRI. The C1s-coding or empty plasmid pcDNA3.1 (Thermo Fisher, cat\# V79520) was amplified by bacteria transformation in E. coli (Thermo Fisher, cat\# C4040-03), cultured in LB agar, supplemented with $100 \mu \mathrm{g} / \mathrm{ml}$ of Ampicillin (Duchefa Biochemie, cat\# A0104.0025), overnight at $37^{\circ} \mathrm{C}$. Clones were selected and amplified in LB medium + Ampicillin. Plasmids were then extracted using ZymoPure Plasmid Maxiprep kit according to the manufacturer's protocol (Zymo Research, cat\# D4202), the gene sequence was then verified (Eurofin Genomics)

\section{Stable transfection in HCT116 cell line}

The transfection was performed in OptiMEM using a mix containing lipofectamine 3000 (dilution 1:100, Thermo Fisher, cat\# L3000015) and the plasmid $(2.5 \mu \mathrm{g} / \mathrm{ml}$, empty or with C1s gene), for $24 \mathrm{~h}$ at $37^{\circ} \mathrm{C}, 5 \% \mathrm{CO}_{2}$. The transfection was stopped by washing and cells were selected using EMEM with Zeocin $(50 \mu \mathrm{g} / \mathrm{ml}$, Thermo Fisher, cat\# R25005). The validation of the transfection was performed by C1s quantification in the supernatant (see ELISA for C1s quantification).

\section{Western blotting}

C1s was evaluated by western blotting using cell culture supernatants or cell lysates. Tumor cell lines, silenced or not for C1s, were cultured in OptiMEM for 48 hours, after which the supernatant was concentrated with Amicon Ultracel 3K units (UFC, cat\# 900324). NuPAGE LDS sample buffer (4X, Thermofisher Scientific, cat\# NP0008) was added to the concentrated supernatants or to the lysate, and the samples were denatured at $80^{\circ} \mathrm{C}$ for 10 minutes. Proteins were separated by NUPAGE $10 \%$ Bis-Tris gel (Thermofisher Scientific) electrophoresis and transferred to a nitrocellulose membrane. The membranes were blocked with $\mathrm{TBS} / 5 \% \mathrm{BSA}$ and incubated overnight on a rocking chair platform with a goat polyclonal antiserum against human $\mathrm{C} 1 \mathrm{~s}$ diluted 1:500 in TBS/5\% BSA. After washing with TBS/0.1\% Tween, an HRP-conjugated rabbit anti-goat IgG (Covalab, cat\# lab0048) was added at 1:1000 dilution for 1 hour at RT under agitation. The chemiluminescent signal was assessed with an iBright CL100 (Thermofisher) after incubation with ECL reagent (Pierce, cat\# 32106).

In addition, a western blot was performed on normal human plasma from 2 healthy donors (diluted 1:10) with the C1s-specific antibody (Abcam, cat\# ab134943) to verify that it recognizes bands corresponding to $\mathrm{C} 1 \mathrm{~s}$ and that there are no non-specific bands. Purified C1s pro-enzyme and enzyme (Complement Technology, cat\# A103 and cat\# A104) were used as positive controls.

MHC class I was detected by western blot on lysate of tumor cells, silenced or not for C1s. NuPAGE LDS sample buffer with reducing agent (DTT, $1 \mathrm{M}$ ) was added and the samples were heated at $80^{\circ} \mathrm{C}$ for $10 \mathrm{~min}$. Proteins were separated on a NuPAGE 10\% Bis-Tris gel. The proteins were transferred onto a nitrocellulose membrane using iBlot (Invitrogen). The membrane was incubated overnight with the primary antibodies (anti-MHC class I A/B/C, Abcam, cat\# 70328) diluted 1:500 in TBS/5\%BSA. After washing with TBS/1\%Tween and incubation for 1 hour with HRP-conjugated goat anti-mouse IgG diluted 1:5000 (Covalab, lab0252), visualization was performed by 
chemiluminescence using a substrate for HRP (SuperSignal ${ }^{\circledR}$ WestDuraLuminol, Thermofischer, cat\# 1856145), detected by iBright Western Blot Imaging System (iBright FL1500 Thermofischer).

\section{qRT-PCR}

Extraction of RNA from silenced cells or cells treated for 48 hours in medium-free serum with IFN $\gamma$ (100 or $1000 \mathrm{ng} / \mathrm{mL}$ ) was performed with a Maxwell cell 16LEV simplyRNA purification kit according to the manufacturer's protocol (Promega, cat\# AS1270). For all conditions, three replicates were included.A 2100 Bioanalyzer (Agilent) and an Agilent RNA 6000 Nano Assay kit (cat\# 5067-1511) were used to determine the quality and quantity of the RNA. Reverse transcription was carried out with the Applied Biosystem High-capacity cDBNA reverse transcription kit according to the manufacturer's protocol (Applied Biosystem, cat\# 4368814). Gene expression of C1S was assessed by Taqman using primers for human C1S (ThermoFisher, cat\# Hs00156159_m1), compared to GAPDH (ThermoFisher, cat\# Hs00266705_g1) and ACTB (ThermoFisher, cat\# Hs01060665_g1) genes for normalization.

\section{Analysis of differentially expressed genes}

For RNASeq analysis, three biological replicates for each condition (siC1s vs siC) were used. The libraries were prepared with NEBNext Ultra II Directional RNA Library Prep Kit (New England BioLab, cat\# E7760) according to the manufacturer's protocol: polyA purification using poly-T oligo attached magnetic beads, followed by 300-bp fragmentation with divalent cations under elevated temperature, synthesis of double-stranded CDNA and amplification by PCR. Sequencing was then carried out using a paired-end 75b of Illumina HiSeq 4000 instrument. The quality of FASTQ files was verified by using FASTQC and then aligned to a reference genome using STAR tool. Once the data were mapped on the genome, HTSeqCount was used to count and assign reads to a given exon and generate a count table. Before differential analysis, non-expressed genes with a count equal to 0 were removed. $\mathrm{R}$ software and the DESeq2 package [18] was used to examine differential expression between siC1s- and siC-transfected cells. Results were considered statistically significant at an adjusted $p$ value of $<0,05$. The top 50 most significantly up- or down-regulated genes were sorted by the adjusted p-value; volcano plots were produced, as was a heatmap using heatmap. 2 and EnhancedVolcano packages in R. Gene Ontology enrichment analysis was performed with WEV-based Gene SeT AnaLysis Toolkit (WebGestalt) with the GO biological process category. The raw data are available under the GEO accession number ID PRJNA718732.

\section{Proliferation and mortality}

Cells were washed with PBS before the addition of CFSE reagent 1:1000 (Invitrogen, cat\# C34554) for 20 minutes at $37^{\circ} \mathrm{C}$. Staining was stopped with the addition of complete culture medium. The cells were then washed and cultured in 6-well plates for 48 hours in the presence of the standard culture 
medium for each cell type. Cell proliferation and mortality were compared in the presence of culture medium with or without 48 hours treatment with C3a, C5a or C1s enzyme or C1s proenzyme (each protein was used at the concentration of $500 \mathrm{ng} / \mathrm{ml}$, protein from Complement technology, cat\# A118, cat\# A144). Supernatants containing dead cells and adherent cells were recovered and stained for DAPI before analysis by flow cytometry (BD Fortessa) (see Flow cytometry).

\section{Cell morphology assessment}

Silenced or control cells were cultured for 24 hours on a round cover glass in the bottom of a 24-well plate in the presence of the standard medium in which the cells were routinely cultured (see Cell lines). After fixation with 4\% PFA for 30 minutes at RT, the cells were stained for the actin cytoskeleton with Phalloidin-IFluor 488 reagent (Abcam, cat\# ab176753) and for nuclei with DAPI. The cover glass was recovered and mounted on a microscope slide with Prolong Glass antifade reagent and scanned with AxioScan (Zeiss).

\section{Migration}

A wound healing assay was performed on a cell monolayer of Caki-1, A498 or HCT116 cells to evaluate migration capacity. The monolayer was obtained when cells reached a confluence of $90 \%$, allowing a good monolayer for the twelve next hours. A scratch was generated with a tip, and photographs were taken under a microscope immediately or after 12 hours. The wound healing tool (http://dev.mri.cnrs.fr/projects/imagej-macros/wiki/Wound Healing Tool) in ImageJ was used to calculate the percentage of recovery. The migration capacity of siC vs siC1s cells was compared in the presence of culture medium with or without C3a, C5a or C1s enzyme or C1s proenzyme. The migration capacity of $\mathrm{siC}$ and siC1s cells was compared in presence of culture medium with or without $24 \mathrm{~h}$ treatment of $\mathrm{C} 3 \mathrm{a}, \mathrm{C} 5 \mathrm{a}$ or $\mathrm{C} 1 \mathrm{~s}$ enzyme or C1s proenzyme (each protein was used at the concentration of $500 \mathrm{ng} / \mathrm{ml}$ ).

\section{Flow cytometry}

Caki-1 cells untreated or siC or siC1s were cultured for 24 hours in the presence or absence of IFN $\gamma$ (500ng/ml). Expression of MHC class I, C3aR and C5aR on tumor cells was studied by flow cytometry. Single-cell suspensions were resuspended in PBS $+10 \%$ heat-inactivated FCS. To block nonspecific antibody binding to Fcy receptors, with Fc block (BD Pharmingen, cat\#564219). Then cells were incubated with a viability marker (Invitrogen, Live/Dead fixable yellow dead cell, cat\# L34968), MHC class I ABC-FITC (BD Pharmingen, cat\# 555552), C3aR-PE (BioLegend, cat\# 345804) and C5aR (BioLegend, cat\# 344310 ) for $30 \mathrm{~min}$ at $4^{\circ} \mathrm{C}$. After a washing step with PBS $+10 \% \mathrm{FCS}$, cells were fixed with a solution of $0.4 \%$ PFA. 
Flow cytometry data were acquired using a BD LSRFortessa X-20 (BD Biosciences) and analysed using FlowJow software. Dead cells were excluded of the analysis. Surface protein expression was quantified by the median fluorescence intensity (MFI).

\section{Co-culture between tumor cells and T cells}

Peripheral blood mononuclear cells (PBMCs) from healthy donors were cultured in RPMI (Gibco, cat\# $61870036)+10 \% \mathrm{FCS}+1 \%$ penicillin and streptomycin, at $37^{\circ} \mathrm{C}, 5 \% \mathrm{CO}_{2}$ in a humidified atmosphere Single-cell suspensions were incubated with $\mathrm{Fc}$ block to prevent non-specific binding to $\mathrm{Fc}$ receptors. Then PBMCs were incubated with a viability marker, CD45-APC Cy7 (Clone 2D1, BD Bioscience, cat\# 561863), CD3-AF700 (Clone UCHT1, BD Bioscience, cat\# 561027), CD4-eF450 (Clone OKT4, eBioscience, cat\# 48-0048-42) and CD8-PerCP Cy5.5 (Clone RPA-T8, eBioscience, cat\# 45-008842). $\mathrm{CD}^{+}$and $\mathrm{CD}^{+} \mathrm{T}$ cells were sorted from PBMCs with a purity superior to $95 \%$ on FACS Aria III cell sorter (BD Bioscience), in RPMI + 10\% FCS. Dead cells were excluded of the experiment, we gated on $\mathrm{CD} 5^{+} \mathrm{CD}^{+}$cells and then selected either $\mathrm{CD}^{+}$cells or $\mathrm{CD}^{+}$cells. After 24 hours of activation by CD3/CD28 beads (Dynabeads Human T-Activator CD3/CD28, Gibco, cat\# 11161D), T cells were seeded in 96-well plates and co-cultured with Caki-1 cells, untreated or siC or siC1s, at ratios 1:1 or 1 T-cell for 5 tumor cells (1:5) and in the presence or absence of plasma-purified C1s enzyme and proenzyme (1:1 mixture, concentration of $500 \mathrm{ng} / \mathrm{ml}$ for each protein). The co-culture was done in RPMI $+10 \%$ FCS $+1 \%$ penicillin and streptomycin. T-cell activation was assessed in each condition by flow cytometry after $24 \mathrm{~h}$ of culture using viability marker, CD45-APC, CD3-AF700, CD4-eF450, CD8-PerCP Cy5.5, CD38 FITC (Clone HIT2, BD Pharmingen, cat\# 555459), CD69-PE (Clone FN50, BD Bioscience, cat\# 555531) and PD-1-APC (Clone EH12.2H7, BioLegend, cat\# 329907). Flow cytometry was performed as described in Flow cytometry. We excluded dead cells from the analyses, gated on the $\mathrm{CD} 45^{+} \mathrm{CD}^{+}$cells, and then either on the $\mathrm{CD} 4^{+}$or $\mathrm{CD}^{+}$cells to study their activation markers. Surface protein expression was quantified by MFI.

\section{Statistical analysis}

The package "survival" in R software was used to generate Kaplan-Meier curves, with censoring at 2500 days. A log-rank test was applied to examine the difference between curves. Association between the distributions of two semi-quantitative variables was assessed by the Chi2 test; association between one semi-quantitative variable and one quantitative variable was evaluated by the Kruskal-Wallis test and correlation between two quantitative variables by the Pearson test. The difference between siC1s and siC cells with regard to proliferation and survival was assessed by the paired T-test; the unpaired T-test is used for cell migration. The Mann-Whitney test was applied to assess the difference in C4d level between ccRCC patients and healthy donors as well as patients with high or low levels of $\mathrm{C} 4 \mathrm{~d}$ deposits inside the tumor core. ${ }^{*} p<0,05, * * p<0,01, * * * p<0,001, * * * *$ $p<0,0001$. 


\section{Bioinformatic analysis}

Bulk RNAseq. C1S gene expression, as measured as transcripts per million (TPM), was determined by using RNAseq data from 28 TCGA cohorts of patients with different cancer types available at cBioPortal [19], [20]. No patients were excluded. In this database, TPM values were generated with the RSEM method (RNA-Seq by Expectation Maximization). Tumor types were arranged according to median expression values. Hepatocellular carcinoma (LIHC) was excluded from the analysis to avoid hiding the potential differences between tumor types linked to the very high expression of complement genes in the liver. Survival analysis was conducted with the Protein Atlas Interactive survival scatter plot \& Survival analysis tool (https://www.proteinatlas.org/) using the optimal cut-off and the Gene Expression Profiling Interactive Analysis (GEPIA) tool (http://gepia.cancer-pku.cn/); the results are represented as the log2 Hazard ratio of $\mathrm{C} 1 \mathrm{~s}$ for overall survival, with an optima cut-off or median cut-off calculated with the $\mathrm{Cox} \mathrm{pH}$ Model respectively. A log-rank test was applied to evaluate differences between the curves; surrounded boxes represent a log-rank p-value $<0,05$. Because of the low number of survival events, the testicular germ cell tumor cohort (TGCT) was also excluded from the survival study. The KIRC cohort of TCGA was used to compare expression $(\log 2(1+T P M))$ of complement genes $(C 1 Q A, C 1 R, C 1 S, C 2, C 3$ and $C 4 A)$ among patients. Heatmaps were generated with $\mathrm{R}$ software 3.4.2 and the heatmap.2 package. Differential expression of $C 1 S$ between tumors versus paired normal tissue from TCGA normal and GTEx normal databases $(n=100)$ were investigated with GEPIA. Due to the absence of normal tissue for uveal melanoma (UVM), this cancer type was not included in the analysis. Kaplan-Meier curves showing the overall survival of patients with high (>median) or low (<median) expression of C1S in different TCGA cohorts were generated with GEPIA. C1s expression in $\log 2(1+$ TPM) in the different TNM stages of ccRCC (KIRC) patients are represented by a violin plot generated with GEPIA. The Pearson coefficient of the correlation between C1s expression (TPM) and that of other complement genes in KIRC are represented as heatmap, as prepared using $\mathrm{R}$ software.

Association between C1S expression in TCGA cCRCC and immune infiltrate genes are based on immune signature of the MCP counter [21].

scRNA-seq. The scRNA-seq count matrix, genes and barcodes files were from Young et al. [22] supplemental data. A total of 2 biopsy samples from one ccRCC patient were used in this study. Seurat (version 3.0.1) [23] was employed for quality control, and single cells with less than $200 \mathrm{UMIs}$ or with more than $25 \%$ mitochondrion-derived UMI counts were considered low-quality cells and removed. Ultimately, 3140 single cells were subjected to further investigation. The FindClusters function (resolution $=1$ ) of Seurat was used to identify cell clusters. The clusters were visualized using 2D uniform manifold approximation and projection (UMAP). The FindAllMarkers function was utilized to list the markers of each cell cluster. The major cell types were then recognized based on the markers obtained from a previous study [24]. The $\mathrm{C}^{+} \mathrm{s}^{+}$cells are defined by a C1S expression level greater than zero. 


\section{$\underline{\text { Results }}$}

\section{Plasma C4d is a non-invasive, negative prognostic factor for ccRCC}

In our retrospective cohort 1 (Supplementary Table S1), C4d deposits conferred poor prognosis [8]. Therefore, we evaluated the level of plasma C4d. It was significantly higher in ccRCC patients than in healthy controls in two independent prospective cohorts, cohort 2 (Figure 1A) and cohort 3 (Figure 1B). The patients had normal C4 levels and no elevated sC5b-9 in plasma.

We observed a trend for patient survival, with progression events only occurring in the group of ccRCC patients in cohort 2 who had plasma C4d levels above normal range (>mean+2SD of the C4d level of healthy controls) (Figure 1C). The difference did not reach statistical significance because of the short follow-up (starting 2017) in this cohort; most patients were free from progression/death at the time of last follow up. In cohort 3, which includes patients with longer follow up (starting in 2014), we observed the negative prognostic impact of high C4d levels on PFS with a cut-off corresponding to the mean+2SD of the simultaneously tested 31 healthy controls (Figure 1D). The increased plasma C4d levels did not correlate with available clinical parameters (sex, Furman grade or TNM stage), immune infiltration or the activation status of the infiltrating immune cells in the tumor (Supplementary Table S3). Patients that displayed local complement activation inside the tumor, as revealed by high level of C4d deposition, had a trend toward increased plasma C4d (Figure 1E). The negative prognostic impact of the $\mathrm{C} 4 \mathrm{~d}$ deposits within the tumor was confirmed in the extended prospective cohort 3 (Figure 1F, Supplementary Figure S1C) of 92 patients for whom we had ccRCC tumor paraffin embedded samples. The C4d deposition was driven mainly by the classical pathway, since we detected colocalization of C1q with C1s (Figure 1G) and C4d with C1s (Figure 1H) deposits at the tumor cell surface. The patients who had both plasma and in situ C4d had the poorest prognosis, although the double negative showed no progression in the studied timeframe (Figure 1I). The patients with elevated C4d in plasma but not in the tumor, had intermediate prognosis; those who had C4d only in the tumor showed no progression. Thus, plasma C4d may be a non-invasive biomarker of poor prognosis in cCRCC.

\section{A subgroup of ccRCC patients displays a complement-rich phenotype}

Local production of complement proteins is key for intratumoral complement activation. In the cCRCC (KIRC) cohort of TCGA, approximately half of the patients displayed a complement-rich tumor environment with simultaneous expression of genes related to the classical complement pathway (C1QA, C1R, C1S, C2, C3 and C4A) (non-supervised classification, Figure 2A). All these genes were expressed at higher levels in ccRCC tumors, compared with normal tissue, according to the TCGA database $\left(p<10^{-10}\right)$. Analysis of the available scRNA-seq dataset of a fresh tumor sample [22] (Figure $2 \mathrm{~B}$ and Supplementary Figure $\mathrm{S} 2$ ) showed that $C 1 S, C 1 R, C 2, C 3$ and some extent $C 1 Q$ genes were expressed in tumor cells but $C 1 Q A$, (also $C 1 Q B, C 1 Q C$ ) and $C 3$ were expressed by macrophages (Figure 2C). Individual tumor cells that expressed $C 1 S$ also expressed $C 1 R$ and $C 2$ and $C 3$ (Figure 2D), 
and this was confirmed for $\mathrm{C} 1 \mathrm{~s}, \mathrm{C} 4$ and $\mathrm{C} 3$ by IF (Figure $2 \mathrm{E}-\mathrm{F}$ ) and IHC (Figure 2G). $\mathrm{C}^{+} \mathrm{s}^{+}$and $\mathrm{C4}^{+}$tumor cells exhibited intermediate/low staining of cytokeratin and C1s+ tumor cells were also vimentin negative, suggesting that they are not mesenchymal cells (Supplementary Figure S3).

We stratified the tumors from the different cohorts according to C1s staining in tumor cells by IHC (Supplementary Figure S4). Most (15/17) of the C1s-high tumors of cohort 1 also expressed high levels of C4 or C3, whereas only half of the C1s-low tumors expressed high levels of C4 or C3 (37/65 and 30/65, respectively). This reflects the positive association between a high density of C1s staining in tumor cells and staining for $C 4(p=0,003)$ and $C 3(p<0,0001)$ (Supplementary Figure S5A-B). An association between local production of $\mathrm{C} 4$ and $\mathrm{C} 1 \mathrm{~s}$ by tumor cells, as determined by $\mathrm{IHC}$, and the presence of $C 4 d$ deposition ( $p=0,038$ and $p=0,017$, respectively) suggested that the local production was in part responsible for complement activation (Supplementary Figure S5C-D).

C1s staining within the tumors was found not only in the tumor cells, but also in some stromal cells (Supplementary Figure S3A). Non-tumoral cells within the TME that expressed C1s included endothelial cells $\left(\mathrm{CD} 31^{+}\right)$and rare macrophages $\left(\mathrm{CD} 68^{+}\right)$(Supplementary Figure $\mathrm{S6}$ ).

\section{C1s predicts a poor prognosis in ccRCC}

Having shown that cCRCC tumor cells produce C1s, we investigated whether it has a prognostic impact by analyzing TCGA data. C1S gene expression was increased in cCRCC tumors compared with normal kidney (Figure 3A), especially in advanced-stage patients) (Figure 3B). C1S gene expression in cCRCC patients from TCGA was associated with a poor prognosis (Figure $3 C$ ). The negative prognostic value of $\mathrm{C} 1 \mathrm{~s}$ was validated at the protein level by IHC in two independent cohorts. A high number of C1s-producing cells (group 2) was associated with poor outcomes in cohort 1 (Figure 3D-E) and cohort 3 (Figure 3F). When patients who were in the C1s-high group were compared with those who were in the C1s-low group (groups 0 and 1)(, the effects on survival were still significant, with decreased survival (OS and PFS) in the high group compared with the low group (Supplementary figure S7A-B) (Cohort 1 PFS $p=0.00403$, OS $p=0.00604$, Cohort 2 PFS $p=4.37 e^{-5}$ ). Like gene expression, a correlation between TNM stage and C1s protein level was observed (Figure 3G). C1s protein also correlated with the presence of advanced Fuhrman grade and metastasis (Figure $3 \mathrm{H}-\mathrm{I}$ and Supplementary Figure S7C). However, in a bivariate Cox model on PFS, C1s retained this independent prognostic value with regard to TNM stage $(p=0.027)$ and metastasis status $(p=0.014)$ but not Fuhrman grade $(p=0.11)$. The negative prognostic impact of $C 1 s$ was observed for stage I and II of the cCRCC cohort in TCGA (PFS $p=0.0084$, Figure $3 \mathrm{~J}$ and $p=0.0074$ Supplementary Figure S7D), but not for the advanced stages III and IV ( $p=0.35$ and $p=0.34)$. The decreased PFS of C1s-high patients with stage I ccRCC was confirmed at the protein level in our cohorts 1 and 3 (PFS $p=0.0042$ and $p=0.0302$ respectively, Figure $3 \mathrm{~K}-\mathrm{L}$ ). Not enough patients were available at stage 2 and no significant difference was found in advanced stages (PFS: stage III $p=0.592$ and stage IV $p=0.226$ for cohort 1 and stage III $p=0.164$ for cohort 3 ). No difference was found also in the JAVELIN cohort of 886 RCC patients with stages III and IV, for whom transcriptomic data is available [25]. The absence of a prognostic value 
of C1s by Kaplan-Meier analysis in advanced stage patients could be related to the strong association between $\mathrm{C} 1 \mathrm{~s}$ expression and TNM stage. This suggests a potential action of $\mathrm{C} 1 \mathrm{~s}$ in the transition from localized to metastatic tumors. Thus, once tumors are already in advanced stage, C1s may no longer be involved.

\section{Canonical mode of action of C1s in the ccRCC tumors}

The most evident mechanism to explain the negative prognostic impact of $\mathrm{C} 1 \mathrm{~s}$ would be its canonical functions in the complement cascade. When the patients were stratified at optimal cut-off and using FPKM (as in Protein Atlas), C5AR1 conferred poor prognosis in cCRCC in the TCGA cohort, whereas C3AR was associated with a favourable outcome (Figure 4A). This significance was lost when the analysis was made with TMP data normalization and therefore should be interpreted with caution. cCRCC tumors are rich in C3AR-expressing macrophages and C5AR1 expressing neutrophils and macrophages, making them responsive to the generated $\mathrm{C} 3 \mathrm{a}$ and $\mathrm{C} 5 \mathrm{a}$ in the tumor microenvironment (Figure 4B).

Indeed, a fraction of the macrophages and neutrophils (Figure $4 C$ ), but to a lesser extent $T$ cells but not B cells (Supplementary Figure S8A) were positive for C5aR1. C1S expression in tumors correlated with macrophage infiltration in the TCGA $c$ CRCC cohort $\left(R=0.45, p=2.2 \times 10^{-16}\right.$, Supplementary Figure $\mathrm{S} 8 \mathrm{~B}$ ) and at the protein level $\left(\mathrm{CD} 163^{+}\right)$in cohort 1 , as measured by IHC (Figure 4D). A composite score reflecting expression of $\mathrm{C} 1 \mathrm{~s}$ and infiltration by $\mathrm{CD} 163^{+}$macrophages revealed a group of high/high patients with a particularly poor outcome (Figure 4E). In TCGA, no correlation was found between $C 1 S$ and neutrophils $(R=0.1 ; p=0.0335$, Supplementary Figure $S 8 C)$.

Other non-tumor cells, which express little if any C3aR1 or C5aR1, also correlated with C1S. A correlation was found with the $B$ cells $\left(R=0.41 ; p<2,2 \times 10^{-16}\right)$, T-cells $\left(R=0.53 ; p<2,2 \times 10^{-16}\right)$ and the fibroblasts $\left(R=0.48 ; p<2,2 \times 10^{-16}\right)$, while $R$ was below 0.3 for the NK cells $\left(R=0.21 ; p=2,843 \times 10^{-6}\right)$ and endothelial cells ( $R=-0.01 ; p=0.03235$ ) (Supplementary Figure S8D-H).

Very few tumor cells expressed C3aR1 or C5aR1 (Figure 4B). Only 1/11 tested tumor samples showed low levels of C5aR1 on tumor cells, although infiltrating stromal cells positive for C5aR1 were abundant (Figure 4F). The ccRCC cell lines Caki-1 and A498 expressed low levels of C3aR and C5aR1 by flow cytometry (Figure $4 G$ for Caki-1) and qRT-PCR. Caki-1 and A498 cells did not generate detectable concentrations of $\mathrm{C} 5 \mathrm{a}$ (detection limit $0.01 \mathrm{ng} / \mathrm{mL}$ ), whereas $\mathrm{C} 3$ a was at the detection limit of the kit, at $0.015 \mathrm{ng} / \mathrm{mL}$ in our experimental conditions. This makes an autocrine effect of C5a less likely. Addition of purified C3a or C5a did not modify the viability, morphology or proliferation of resting Caki-1 cells (Figure 4H-J). Silencing of $\mathrm{C} 1 \mathrm{~s}$ also did not make the Caki-1 cells proliferation sensitive to C3a or C5a. C3a did reduce the migration capacity of Caki-1 cells, but C5a had no effect on this (Figure $4 \mathrm{~K}-\mathrm{L}$ ). Taken together, these results suggest that $\mathrm{C} 3 \mathrm{a}$, but not $\mathrm{C} 5 \mathrm{a}$, may have an effect on tumor cells. The generation of $\mathrm{C} 3 \mathrm{a}$ and $\mathrm{C} 5 \mathrm{a}$ by the canonical role of $\mathrm{C} 1 \mathrm{~s}$ may result in remodelling of the TME. 
Cox multivariate analysis in cohort 1 highlighted that the prognostic value of $\mathrm{C} 1 \mathrm{~s}$ was independent of complement activation markers ( $C 1 q$ deposits $(p=0.00148)$, C $4 d$ deposits $(p=0.00741)$ and $C 3 d$ deposits $(p=0.00488))$, suggesting a potential cascade-independent role for $\mathrm{C} 1 \mathrm{~s}$.

\section{C1s modulates the transcriptional program of ccRCC tumor cells}

Considering that the prognostic value of C1s may be independent of complement activation, we investigated $\mathrm{C} 1 \mathrm{~s}$ effects on tumor cells. At the single-cell level (in a dataset from a fresh ccRCC human tumor [22]), we searched for genes that discriminated tumor cells expressing C1S from those that did not (Figure 5A). Gene Ontology analysis showed that genes enriched in $\mathrm{C}_{1}{ }^{+}$tumor cells are implicated in various pathways such as vesicle mediated transport, response to oxygen-containing compounds, response to hormones, myeloid activation, neutrophil activation and degranulation, response to endoplasmic reticulum stress, extracellular matrix organization, complement proteins, regulation of IGF transport and uptake, and folding assembly and peptide loading of MHC class I (Figure 5B-C). To study the direct effects of $\mathrm{C} 1 \mathrm{~s}$ on tumor cells, we performed in vitro studies on Caki1 and A498 cells with or without C1S silencing. Both cell lines produced and secreted the pro-enzyme and enzyme forms of C1s (Supplementary Figure S9A-B). After validation of the efficacy of C1S silencing in Caki-1 and A498 cells by qRT-PCR and western blotting (Supplementary figure S9C-D and S9E-F, respectively), we performed RNA-Seq for Caki-1 (Figure 5D) and A498 (Supplementary figure S10A) cells. Among all genes, we identified 56 downregulated genes and 119 upregulated genes in Caki-1 siC1s vs. siC cells and 41 downregulated genes and 112 upregulated genes in A498 siC1s vs. siC cells by considering a fold change $>2$ in absolute value and an adjusted $p$-value $<0,05$ (Figure 5E and Supplementary figure S10B). Enrichment analysis with GO:biological process and Reactome highlighted that these genes are involved in different pathways, some previously highlighted by the single-cell analysis (Figure 4F-G and Supplementary figure S10C-D). Among the upregulated pathways in siC1s Caki-1 cells, we found regulation of development and growth, regulation of chemotaxis, and extracellular matrix organization. Downregulated genes were implicated in extracellular matrix organization, IGF transport and uptake, and endosomal/vacuolar pathway. For A498 cells, we observed upregulation of genes involved in regulation of motility, extracellular matrix organization and cellular response to erythropoietin as well as downregulation of genes involved in endocytosis, vesicle mediated transport, angiogenesis and transcriptional regulation by RUNX1.

\section{C1s modulates the proliferation and survival of renal and lung tumor cells}

In culture, we observed visual phenotypic changes in siC1s cells, with a longer time to reach confluence. Functionally, silencing of C1S in Caki-1 and A498 cells induced a decrease in viability (Figure 6A and Supplementary S10E) and proliferation capacity (Figure 6B-C and Supplementary S10F-G) but no effect on cell morphology (Figure 6D and Supplementary S10H) or migration capacity (Supplementary figure S10I-J). 
Expression of $\mathrm{C} 1 \mathrm{~s}$ by tumor cells was not limited to ccRCC. Analysis of C1S expression in 28 cohorts of the PanCancer study of TCGA [26] revealed heterogeneous expression according to tumor type (Supplementary Figure S11A). The almost 100-fold higher expression of C1S in pancreatic adenocarcinoma and sarcoma than in kidney chromophobe represents the two extremes. NSCLC subtypes lung adenocarcinoma and lung squamous cell carcinoma were among the cancer types in which C1S was strongly expressed. We also found that a fraction of the cells (defined by CK staining) in an NSCLC tumor were positive for C1s protein (Supplementary figure S11B-C). Moreover, silencing of C1S affected the phenotype of the lung cancer cell line A549 in similar ways to that seen for the ccRCC cell lines (Supplementary figure S11D-E).

\section{C1s in the extracellular milieu does not impact the phenotype of cancer cells}

To find out if the phenotypic alterations of the siC1s Caki-1 cells could be mediated by the absence of secreted C1s, we added purified C1s pro-enzyme or enzyme in the supernatant. No effect on the proliferation and viability was detected (Figure 6E). Further, we attempted to "transplant" the phenotype of enhanced proliferation to a naturally C1s-deficient cell line, HCT116, by transfecting it with a C1S-expressing plasmid (Supplementary Figure S12). The recombinant C1s was efficiently expressed, but it was entirely released in the supernatant, without intracellular retention (Supplementary Figure S12A-B). Two C1s-expressing clones were selected and both showed no difference in proliferation and migration compared with untreated cells or cells transfected with empty vector (Supplementary Figure S12C-E).

\section{C1s interplay with the T-cells}

C1s expression in fibroblasts is induced by IFN $\gamma$ [27], produced by tumor-infiltrating T-cells. We found that IFN $\gamma$ stimulated C1S expression in ccRCC tumor cell lines in vitro (Figure 7A and Supplementary Figure S13). Expression of IFNG and C1S correlated positively in the cCRCC cohort of TCGA $(R=0.26$, $\left.\mathrm{p}=1.5 \times 10^{-9}\right)$. One of the main target genes of IFN $\gamma$ in tumor encodes MHC class I. C1s was suggested to cleave MHC class I but the C1S silencing did not modify the protein integrity of MHC class I, as detected by western blot on Caki-1 lysates (Supplementary Figure S14A). Exposure of Caki-1 cells to IFN $\gamma$ resulted in upregulation of MHC class I, as detected by flow cytometry (Figure 7B-C and Supplementary Figure S14B-C). The siC1s Caki-1 showed a trend toward upregulation of MHC class I at basal level (Figure 7B). This increase is also observed upon IFN $\gamma$ stimulation, but a clear alteration of the expression pattern was detected, with robust appearance of two populations with different granularity (Figure 7C and Supplementary Figure S14C), which requires further study.

This difference in surface expression of MHC class I was not shared by other surface markers, since C3aR and C5aR1, detected in triple staining on the same cells did not show any alteration (Figure 4G). No significant differences in the patterns of MHC class I staining on C1s-high or C1s-low ccRCC tumors was observed (Supplementary Figure S14D). 
T cells are a major source of IFN $\gamma$ in the TME [28], [29]. In ccRCC the high CD8 ${ }^{+}$T-cell density is associated with poor prognosis [17], [30], as confirmed in cohort 1 (Figure 7D). A previously defined $\mathrm{CD}^{+} \mathrm{T}$-cell signature [21] correlated of $\mathrm{C} 1 \mathrm{~S}$ with $\mathrm{CD} 8^{+} \mathrm{T}$ cells $(\mathrm{R}=0.42, \mathrm{p}=<2.2 \mathrm{e}-16$, Figure 7E). The expression of $C 1 S$ correlated with $C D 4\left(R=0.35, p=1.5 \times 10^{-16}\right)$. Furthermore, the number of $\mathrm{C}^{+} \mathrm{s}^{+}$cells within the tumor correlated with increased $\mathrm{CD} 8^{+} \mathrm{T}$-cell infiltration (Figure7F) and PD1 expression (Figure 7G) as detected by IHC in cohort 1 . In this cohort the high density of $\mathrm{CD} 8^{+} \mathrm{T}$ cells was associated with poor prognosis (PFS: $p=0.0363$; OS: $p=0.0192$ ). For 34 patients from cohorts 2 and 3 for whom RNAseq data was available, C1S also correlated with IFNG $(R=0.366, p=0.0281)$ and showed a trend for $C D 8 B(R=0.31, p=0.0694)$.

To find out whether tumor cell-derived C1s could impact the T cells, a co-culture was set up between untreated, siC or siC1s Caki-1 cells and resting or activated sorted $\mathrm{CD} 4^{+}$and $\mathrm{CD} 8^{+} \mathrm{T}$ cells.. In contrast, incubation with siC1s cells induced further activation of pre-stimulated T cells, measured by increase in the \% of $\mathrm{CD}_{3} 8^{+} \mathrm{CD} 4^{+}$and $\mathrm{CD} 8^{+}$T cells (Figure $7 \mathrm{H}, \mathrm{K}$ ), without modification of the \% of $\mathrm{CD} 69^{+}$or PD$1^{+}$(Supplementary Figure S15). These effects were dose-dependent and increased with the proportion of tumor cells in the co-culture (0:1; 1:1 and 5:1 tumor cells to T cells ratio, Figure 7I,L). Purified C1s enzyme + pro-enzyme had no effect on the activation status of the T cells, compared with albumin and could not revert the phenotype induced by the silencing of C1s (Figure 7J,M). Prestimulated T cells exposed to purified C1s, C3a or C5a did not show alteration of the expression of CD38, CD69 or PD1. Since the experiments were not performed with antigen-specific $T$ cells, the observed increase in T-cell activation may be due to a different expression of co-stimulating molecules or soluble mediators by the tumor cells after $\mathrm{C} 1 \mathrm{~s}$ silencing. These data suggest that the effect is mediated by intrinsic (intracellular) C1s.

No correlation between immune markers and $C 4 d$ deposits $(p=0.22$ for $C D 8, p=0.2$ for PD1 and $p=0.48$ for CD163), plasma C4d (see Supplementary table S3), or C3d deposits ( $p=0.9$ for CD8, $p=0.35$ for PD-1 and $p=0.56$ for CD163) was found. This also suggests another, potentially complementindependent role for $\mathrm{C} 1 \mathrm{~s}$ in the modulation of the TME.

\section{Discussion}

Here, we describe a complement-rich phenotype for $\mathrm{ccRCC}$ in which overexpression of C1s confers a poor prognosis in early stage disease. The associated pro-tumor impact is driven in part by canonical complement system-related inflammation, as reflected by an elevation in plasma $\mathrm{C} 4 \mathrm{~d}$, which has the potential to be a non-invasive, negative prognostic biomarker for ccRCC. Furthermore, we discovered non-canonical, cascade-unrelated, intracellular functions for $\mathrm{C} 1 \mathrm{~s}$ that affect cell transcriptional activity, proliferation, viability and cross-talk with T cells. The joint action of these mechanisms might explain the negative prognostic impact of $\mathrm{C} 1 \mathrm{~s}$ in $\mathrm{ccRCC}$ (Figure $7 \mathrm{~N}$ ), which appears to be a prototypical complement-dependent cancer.

ccRCC is a cancer type in which concomitant overexpression of complement genes confers poor prognosis[2]. Although $\mathrm{C} 1 \mathrm{q}$ originates from macrophages, the remaining proteins are produced by tumor cells. The production of complement proteins within the tumor is heterogeneous, but we 
found that in most cases, the same tumor cell produces $\mathrm{C} 1 \mathrm{~s}, \mathrm{C} 1 \mathrm{r}, \mathrm{C} 2, \mathrm{C} 4$ and $\mathrm{C} 3$; we termed this a complement-rich phenotype. These data support the notion that in a subset of ccRCC tumors the classical pathway components are activated locally [31], [32].

In searching for reliable, non-invasive complement-related biomarkers that could predict patient outcome, we found that plasma C4d was significantly increased in the plasma of ccRCC patients and conferred a poor prognosis. This result, together with similar findings in lung cancer [10] and malignant pleural mesothelioma [11], make plasma $\mathrm{C} 4 \mathrm{~d}$ a novel promising parameter for evaluating the prognosis of cancer patients. Such a rapid screening test has already been used in some patients to predict recurrence of lupus nephritis [33]. Upon further validation, it might be possible for expert complement laboratories to introduce such a test into the routine screening of ccRCC and lung cancer patients. The origin of plasma $\mathrm{C} 4 \mathrm{~d}$ requires further investigation. No hypocomplementaemia was present in any of the patients evaluated, suggesting no consumption of $\mathrm{C} 4$ in the circulation. $\mathrm{C} 4 \mathrm{~d}$ can be generated via the classical or the lectin pathway, but both require a multivalent ligand on a surface for activation [4]. Therefore, plasma C4d likely derives from the tumor. Consistent with this, we detected a trend toward an association between $\mathrm{C} 4 \mathrm{~d}$ deposits in the tumor and in the plasma. Thus, plasma C4d might arise due to unsuccessful binding of $\mathrm{C} 4 \mathrm{~b}$ to tumor cells or proteolytic shedding of the $\mathrm{C} 4$ bound target and subsequent inactivation by Factor I or due to release as a part of membrane-bound $\mathrm{C} 4 \mathrm{~d}$ through microvesicles. Such a process of $\mathrm{C} 4 \mathrm{~d}$ elimination via microvesicles is described in renal transplantation [34] but remains to be examined in cancer.

Intratumor complement activation allows the release of $\mathrm{C} 5 \mathrm{a}$, generating cancer-promoting signals through C5aR1 [2], [8], [31], [34], [35]. At optimal cut-off, C5AR1 gene expression was associated with poor prognosis, but it is difficult at this stage to conclude which cells this negative prognostic impact is due to, i.e. immune cells and/or tumor cells. This is due to doubts on the specificity of the S5/1 and P12/1 C5aR1-specific antibody clones [37], which were used to detect C5aR1 in renal cancer [32], [35], [38]. As in [37], we used another clone, 8D6, and found few tumor cells positive for C5aR1 by IHC in agreement with the ScRNAseq. The ccRCC tumor cell lines tested here did not generate detectable $\mathrm{C} 5 \mathrm{a}$ in the supernatant, arguing against an autocrine effect in the absence of canonical complement activation. Although Caki-1 cells expressed low level of C5aR1 on the surface, there were no changes in morphology, proliferation or migration in response to C5a. The most likely effect of $\mathrm{C5a}$ is, therefore, on the immune infiltrate, attracting and activating tumor-associated macrophages and neutrophils.

C3AR gene expression at optimal cut-off was associated with favorable outcome in ccRCC. We detected C3aR on the tumor cell lines, and Caki-1 cells had reduced migration capacity in respose to C3a. Expression of the $\mathrm{C} 3$ gene and the intracellular presence of $\mathrm{C} 3$ protein conferred poor prognosis in ccRCC, but deposits of C3d did not [8]. The intracellular Factor $\mathrm{H}$ staining partially colocalized with C3 and also conferred poor prognosis in ccRCC [39]. Together, these results hint to a peculiar, dual action for $\mathrm{C} 3$ in $\mathrm{CCRCC}$, a protective one via $\mathrm{C3}$ a and a deleterious one, potentially via progression of the cascade to $\mathrm{C} 5 \mathrm{a}$ (without formation of $\mathrm{C} 5 \mathrm{~b}-9$ ) and likely by a novel, intracellular mode of action, similar to what is already described in T cells [40] and pancreatic islets [41], [42], which may be in part linked to Factor $\mathrm{H}$. 
In this study we describe an intracellular, non-canonical mode of action for C1s in tumor cells. Multivariate Cox analysis revealed the negative prognostic value of $\mathrm{C} 1 \mathrm{~s}$ to be independent of other complement activation markers such as $\mathrm{C} 1 \mathrm{q}$, and $\mathrm{C} 4 \mathrm{~d}$ and $\mathrm{C} 3 \mathrm{~d}$ deposits, highlighting a cascadeindependent role for C1s. C1S silencing altered the transcriptional program of ccRCC cells and reduced cell proliferation and viability. This phenotype was independent of $V H L$ mutation, as it was observed in Caki-1 cells, which are VHL wild-type and A498 cells, which are VHL mutated. Noncanonical functions for $\mathrm{C} 1 \mathrm{~s}$ have also been reported in CSCC, although the genes differentially expressed after C1S silencing were different [15]. Moreover, reduced proliferation and viability were observed in the lung adenocarcinoma cell line A549, suggesting the general relevance of C1s as a proliferation- and cell survival-promoting factor.

Some studies suggest that C1s can cleave substrates other than C4 [43]-[48], [48]-[50], forming a C1s substratome. Therefore, we hypothesized that upon secretion, C1s can cleave a specific protein(s) from the extracellular space or at the cell surface that will allow an increase in proliferation or survival. C1s was reported to degrade components of the extracellular matrix such as decorin, type 1 gelatine and type 2 collagens [49], MHC class I [51] and insulin-like growth factor binding protein 5 (IGFBP5) [46]. We did not detect MHC class I cleavage, collagen degradation or IGFR signalling. Moreover, addition of purified C1s pro-enzyme or enzyme in the culture medium failed to prevent the effect of the C1S gene silencing on proliferation, survival and T-cell activation. Secretion of recombinant $\mathrm{C} 1 \mathrm{~s}$ from HCT116 cells also had no measurable effect on their phenotype. Therefore, the extracellular activity of $\mathrm{C} 1 \mathrm{~s}$ does not seem to play a detectable role in our experimental conditions and cannot explain the observed phenotype.

The subcellular localization of $\mathrm{C} 1 \mathrm{~s}$ in the cytoplasm hints at potential mechanisms by which it can affect fundamental cellular functions. C1s interacts and cleaves HMGB-1 [52], [53], which shuttles between the cytoplasm and the nucleus [54]. In the nucleus, HMGB1 interacts with nucleosomes, transcription factors, and histones, regulating transcription. Therefore, C1s may affect transcription via its interaction/cleavage of HMGB-1. This may explain the link between C1s expression and the enrichment in genes related to extracellular matrix organization, regulation of IGF transport and uptake by insulin-like growth factor binding proteins or vesicular transport, as evidenced by scRNAseq of a ccRCC patient and RNAseq of siC1s cells.

Intracellular cleavage of C3 has a pro-survival role in T cells [40]. C1s is co-expressed with other complement proteins, such as $\mathrm{C} 1 \mathrm{r}, \mathrm{C} 2, \mathrm{C} 3$ and $\mathrm{C} 4$ in the context of the complement-rich phenotype of the tumor cells. Therefore, intracellular C1s may get activated and exert enzymatic activity toward its canonical substrates but in a non-canonical location. Therefore, the activity of C1s may result in functions, similar to the ones described for $\mathrm{C} 3$ in T cells [55].

A fraction of the C1s-high tumors is rich in $\mathrm{T}$ cells, as seen here and in a recent unsupervised transcriptomic analysis 823 RCC patients [56], suggesting an interplay between these two factors. Pro-inflammatory cytokines such as IFN $\gamma$ produced by T cells stimulate expression of C1S and other complement genes in many cells[27]'[57]'[58], including the ccRCC cell lines used in our study. CD8 ${ }^{+} T$ cells and IFN $\gamma$ confer poor prognosis in ccRCC [17], [30]. The increased $\mathrm{CD} 4^{+}$and $\mathrm{CD} 8^{+} \mathrm{T}$-cell activation occurred without concomitant PD1 increase when in contact with C1s-silenced tumor cells. These 
data suggest a bi-directional cross-talk in C1s-high/T-cell-high tumors whereby infiltrating $\mathrm{T}$ cells stimulate C1s via IFN $\gamma$ and C1s-high tumor cells prevent T-cell activation and likely promote exhaustion, as the amount of $\mathrm{PD}^{+}$cells also correlated with $\mathrm{C} 1 \mathrm{~s}$ in situ. This cross-talk may be related to the phenotype of the tumor cells (expressing C1s or not) and not to the C1s in the surrounding milieu, again pointing toward an intracellular, non-canonical mode of action for C1s.

The action of $\mathrm{C} 1 \mathrm{~s}$ is context dependent. $\mathrm{C} 1 \mathrm{~s}$ is associated with deleterious clinicopathological parameters in urothelial carcinoma [12] and CSCC [59], similar to our findings for ccRCC. Nevertheless, C1s overexpression in sarcomas confers a favourable outcome [2]. Therefore, the mechanisms of action of complement in cancer are complex and context dependent and must be experimentally explored in every particular context.

In conclusion, local synthesis of complement proteins allows activation of the classical complement pathway, that correlates with decreased survival. Moreover, C1s promotes tumor growth both by canonical classical pathway activation and a non-canonical, intracellular mechanism resulting in enhancement of tumor cell proliferation and modulation of the TME. These mechanisms likely synergize to confer a poor prognosis. Overexpression of $\mathrm{C} 1 \mathrm{~s}$ by tumor cells could be a new escape mechanism to promote tumor progression.

\section{Acknowledgements}

We are grateful to Veronique Rossi and Isabelle Bally (IBS, Grenoble) for providing the pcDNA3.1 C1sexpressing vector, described in [52].

We are grateful to the team of the platform "Centre d'Histologie, d'Imagerie et de Cytometrie», (CHIC) at our Centre de Recherche des Cordeliers UMRS1138 (Paris, France) for the technical assistance. CHIC is a member of the Sorbonne University Flow Cytometry Network (RECYF). We thank Isabelle Sauret and Simon Lefranc from"Centre de Ressources Biologiques" and the Pathology Department at the IMM for their help in the sample storage and collection.

\section{Authors contribution}

Designed the study: LTR, MVD, IC, CSF, WHF. Performed research: MVD, MR, JR, MADD, TRR, VP, AG, $G L, A B, M M$. Analysed and discussed the data: MVD, MR, JR, MADD, CG, TRR, VP, AG, GL, AB, MM, VFB, IC, DD, CSF, WHF, LTR. Provided patients samples and take care of the patients: VV, SMO, AM, YAV, GP, PV, XC, RSS, DD. Wrote the manuscript: MVD, MR, LTR. All authors read and approved the submission.

\section{$\underline{\text { References }}$}


[1] G. Hajishengallis, E. S. Reis, D. C. Mastellos, D. Ricklin, et J. D. Lambris, « Novel mechanisms and functions of complement ", Nat. Immunol., vol. 18, n 12 , p. 1288-1298, nov. 2017, doi: 10.1038/ni.3858.

[2] L. Roumenina, M. V. Daugan, F. Petitprez, C. Sautès-Fridman, et W. H. Fridman, " Contextdependent roles of complement in cancer ", Nat. Rev. Cancer, 2019.

[3] C. Gaboriaud, W. L. Ling, N. M. Thielens, I. Bally, et V. Rossi, « Deciphering the fine details of c1 assembly and activation mechanisms: "mission impossible"? ", Front. Immunol., vol. 5, p. 565, 2014, doi: 10.3389/fimmu.2014.00565.

[4] N. S. Merle, S. E. Church, V. Fremeaux-Bacchi, et L. T. Roumenina, "Complement System Part I Molecular Mechanisms of Activation and Regulation », Front. Immunol., vol. 6, p. 262, 2015, doi: 10.3389/fimmu.2015.00262.

[5] I. Bally et al., "Interaction of C1q With Pentraxin 3 and IgM Revisited: Mutational Studies With Recombinant C1q Variants ", Front. Immunol., vol. 10, p. 461, 2019, doi: 10.3389/fimmu.2019.00461.

[6] T. H. Sharp, A. L. Boyle, C. A. Diebolder, A. Kros, A. J. Koster, et P. Gros, « Insights into IgMmediated complement activation based on in situ structures of IgM-C1-C4b », Proc. Natl. Acad. Sci. U. S. A., vol. 116, $n^{\circ} 24$, p. 11900-11905, juin 2019, doi: 10.1073/pnas.1901841116.

[7] D. Ugurlar et al., « Structures of C1-IgG1 provide insights into how danger pattern recognition activates complement ", Science, vol. 359, $n^{\circ} 6377$, p. 794-797, févr. 2018, doi: $10.1126 /$ science.aao4988.

[8] L. T. Roumenina et al., "Tumor Cells Hijack Macrophage-Produced Complement C1q to Promote Tumor Growth », Cancer Immunol. Res., juin 2019, doi: 10.1158/2326-6066.CIR-180891.

[9] D. Ajona et al., « Investigation of Complement Activation Product C4d as a Diagnostic and Prognostic Biomarker for Lung Cancer ", JNCI J. Natl. Cancer Inst., vol. 105, n 18, p. 1385-1393, sept. 2013, doi: 10.1093/jnci/djt205.

[10] D. Ajona et al., " Complement C4d-specific antibodies for the diagnosis of lung cancer ", Oncotarget, vol. 9, $n^{\circ}$ 5, p. 6346-6355, déc. 2017, doi: 10.18632/oncotarget.23690.

[11] T. Klikovits et al., " Circulating complement component 4d (C4d) correlates with tumor volume, chemotherapeutic response and survival in patients with malignant pleural mesothelioma ", Sci. Rep., vol. 7, $n^{\circ} 1$, Art. $n^{\circ} 1$, nov. 2017, doi: 10.1038/s41598-017-16551-7.

[12] I.-W. Chang et al., "Complement Component 1, s Subcomponent Overexpression is an Independent Poor Prognostic Indicator in Patients with Urothelial Carcinomas of the Upper Urinary Tract and Urinary Bladder ", J. Cancer, vol. 7, n 11, p. 1396-1405, juin 2016, doi: 10.7150/jca.15339.

[13] N. Sakai, M. Kusunoki, M. Nishida, T. Toyoguchi, H. Fukutomi, et H. Sakiyama, " Tumorigenicity of BALB3T3 A31 cells transfected with hamster-complement-C1s cDNA ", Int. J. Cancer, vol. 58, $\mathrm{n}^{\circ} 2$, p. 309-313, juill. 1994.

[14] H. Sakiyama et al., "Site-directed mutagenesis of hamster complement C1S: Characterization with an active form-specific antibody and possible involvement of C1S in tumorigenicity ", Int. J. Cancer, vol. 66, n 6, p. 768-771, 1996, doi: 10.1002/(SICI)1097-0215(19960611)66:6<768::AIDIJC10>3.0.CO;2-\#.

[15] P. Riihilä et al., "Tumor cell-derived complement components C1r and C1s promote growth of cutaneous squamous cell carcinoma ", Br. J. Dermatol., mai 2019, doi: 10.1111/bjd.18095.

[16] K. Yu et al., " Construction of a competitive endogenous RNA network and analysis of potential regulatory axis targets in glioblastoma ", Cancer Cell Int., vol. 21, n 1, p. 102, févr. 2021, doi: 10.1186/s12935-021-01789-z.

[17] N. A. Giraldo et al., "Orchestration and Prognostic Significance of Immune Checkpoints in the Microenvironment of Primary and Metastatic Renal Cell Cancer ", Clin. Cancer Res. Off. J. Am. Assoc. Cancer Res., vol. 21, n 13, p. 3031-3040, juill. 2015, doi: 10.1158/1078-0432.CCR-142926. 
[18] M. I. Love, W. Huber, et S. Anders, « Moderated estimation of fold change and dispersion for RNA-seq data with DESeq2 », Genome Biol., vol. 15, n²12, p. 550, déc. 2014, doi: 10.1186/s13059-014-0550-8.

[19] E. Cerami et al., " The cBio cancer genomics portal: an open platform for exploring multidimensional cancer genomics data ", Cancer Discov., vol. 2, n 5, p. 401-404, mai 2012, doi: 10.1158/2159-8290.CD-12-0095.

[20] J. Gao et al., «Integrative analysis of complex cancer genomics and clinical profiles using the cBioPortal ", Sci. Signal., vol. 6, n² 269, p. pl1, avr. 2013, doi: 10.1126/scisignal.2004088.

[21] E. Becht et al., "Estimating the population abundance of tissue-infiltrating immune and stromal cell populations using gene expression », Genome Biol., vol. 17, $n^{\circ} 1$, p. 218, oct. 2016, doi: 10.1186/s13059-016-1070-5.

[22] M. D. Young et al., " Single-cell transcriptomes from human kidneys reveal the cellular identity of renal tumors ", Science, vol. 361, n 6402 , p. 594-599, août 2018, doi: 10.1126/science.aat1699.

[23] T. Stuart et al., « Comprehensive Integration of Single-Cell Data », Cell, vol. 177, n ${ }^{\circ}$ 7, p. 18881902.e21, juin 2019, doi: 10.1016/j.cell.2019.05.031.

[24] J. Hu et al., " Single-Cell Transcriptome Analysis Reveals Intratumoral Heterogeneity in ccRCC, which Results in Different Clinical Outcomes ", Mol. Ther., vol. 0, $n^{\circ}$ 0, avr. 2020, doi: 10.1016/j.ymthe.2020.04.023.

[25] R. J. Motzer et al., " Avelumab plus axitinib versus sunitinib in advanced renal cell carcinoma: biomarker analysis of the phase 3 JAVELIN Renal 101 trial ", Nat. Med., vol. 26, $n^{\circ} 11, p$. 1733-1741, nov. 2020, doi: 10.1038/s41591-020-1044-8.

[26] K. A. Hoadley et al., "Cell-of-Origin Patterns Dominate the Molecular Classification of 10,000 Tumors from 33 Types of Cancer ", Cell, vol. 173, n² 2, p. 291-304.e6, avr. 2018, doi: 10.1016/j.cell.2018.03.022.

[27] Y. Katz et R. C. Strunk, "Synthesis and regulation of C1 inhibitor in human skin fibroblasts ", J. Immunol. Baltim. Md 1950, vol. 142, nº, p. 2041-2045, mars 1989.

[28] T. K et al., " IFN- $\gamma$ is required for cytotoxic T cell-dependent cancer genome immunoediting. ", Nat. Commun., vol. 8, p. 14607-14607, févr. 2017, doi: 10.1038/ncomms14607.

[29] J. R. Schoenborn et C. B. Wilson, « Regulation of interferon-gamma during innate and adaptive immune responses ", Adv. Immunol., vol. 96, p. 41-101, 2007, doi: 10.1016/S00652776(07)96002-2.

[30] W. H. Fridman, L. Zitvogel, C. Sautès-Fridman, et G. Kroemer, "The immune contexture in cancer prognosis and treatment ", Nat. Rev. Clin. Oncol., vol. 14, n ${ }^{\circ} 12$, Art. $n^{\circ} 12$, déc. 2017, doi: 10.1038/nrclinonc.2017.101.

[31] B. Reese et al., " Complement as Prognostic Biomarker and Potential Therapeutic Target in Renal Cell Carcinoma ", J. Immunol. Baltim. Md 1950, vol. 205, n 11, p. 3218-3229, déc. 2020, doi: $10.4049 / \mathrm{jimmunol} .2000511$.

[32] G. S. Netti et al., " PTX3 modulates the immunoflogosis in tumor microenvironment and is a prognostic factor for patients with clear cell renal cell carcinoma ", Aging, vol. 12, $n^{\circ} 8, p$. 7585-7602, avr. 2020, doi: 10.18632/aging.103169.

[33] M. Martin et al., " Plasma C4d as marker for lupus nephritis in systemic lupus erythematosus ", Arthritis Res. Ther., vol. 19, n 1, p. 266, déc. 2017, doi: 10.1186/s13075-017-1470-2.

[34] C. M. Tower et al., " Plasma C4d+Endothelial Microvesicles Increase in Acute AntibodyMediated Rejection ", Transplantation, vol. 101, n 9, p. 2235-2243, 2017, doi: 10.1097/TP.0000000000001572.

[35] W. Xi et al., « Enrichment of C5a-C5aR axis predicts poor postoperative prognosis of patients with clear cell renal cell carcinoma ", Oncotarget, vol. 7, $n^{\circ}$ 49, p. 80925-80934, déc. 2016, doi: 10.18632/oncotarget.13108.

[36] W. Xi et al., " High Level of Anaphylatoxin C5a Predicts Poor Clinical Outcome in Patients with Clear Cell Renal Cell Carcinoma », Sci. Rep., vol. 6, p. 29177, 06 2016, doi: 10.1038/srep29177. 
[37] Z. Kiafard, T. Tschernig, S. Schweyer, A. Bley, D. Neumann, et J. Zwirner, « Use of monoclonal antibodies to assess expression of anaphylatoxin receptors in tubular epithelial cells of human, murine and rat kidneys ", Immunobiology, vol. 212, n² 2, p. 129-139, 2007, doi: 10.1016/j.imbio.2006.11.003.

[38] Y. Maeda et al., " C5aR is frequently expressed in metastatic renal cell carcinoma and plays a crucial role in cell invasion via the ERK and PI3 kinase pathways ", Oncol. Rep., vol. 33, n 4, p. 1844-1850, avr. 2015, doi: 10.3892/or.2015.3800.

[39] M. V. Daugan et al., "Intracellular Factor H drives tumor progression independently of the complement cascade ", Cancer Immunol. Res., 2021.

[40] M. K. Liszewski et al., "Intracellular complement activation sustains T cell homeostasis and mediates effector differentiation ", Immunity, vol. 39, $n^{\circ}$ 6, p. 1143-1157, déc. 2013, doi: 10.1016/j.immuni.2013.10.018.

[41] B. C. King et al., "Complement Component C3 Is Highly Expressed in Human Pancreatic Islets and Prevents $\beta$ Cell Death via ATG16L1 Interaction and Autophagy Regulation ", Cell Metab., vol. 29, n 1, p. 202-210.e6, 08 2019, doi: 10.1016/j.cmet.2018.09.009.

[42] B. C. King, E. Renström, et A. M. Blom, « Intracellular cytosolic complement component C3 regulates cytoprotective autophagy in pancreatic beta cells by interaction with ATG16L1 ", Autophagy, vol. 15, n 5, p. 919-921, mai 2019, doi: 10.1080/15548627.2019.1580515.

[43] J. G. Yeo et al., "Proteolytic inactivation of nuclear alarmin high-mobility group box 1 by complement protease C1s during apoptosis ", Cell Death Discov., vol. 2, p. 16069, sept. 2016, doi: $10.1038 /$ cddiscovery.2016.69.

[44] A. T. Naito et al., " Complement C1q activates canonical Wnt signaling and promotes agingrelated phenotypes ", Cell, vol. 149, n 6, p. 1298-1313, juin 2012, doi: 10.1016/j.cell.2012.03.047.

[45] H. Sakiyama, K. Kaji, K. Nakagawa, et K. Nagino, « Inhibition of bFGF activity by complement C1s: covalent binding of C1s with bFGF », Cell Biochem. Funct., vol. 16, ${ }^{\circ} 3$, p. 159-163, sept. 1998, doi: 10.1002/(SICI)1099-0844(199809)16:3<159::AID-CBF779>3.0.CO;2-8.

[46] W. H. Busby, T. J. Nam, A. Moralez, C. Smith, M. Jennings, et D. R. Clemmons, "The complement component $\mathrm{C} 1 \mathrm{~s}$ is the protease that accounts for cleavage of insulin-like growth factor-binding protein-5 in fibroblast medium ", J. Biol. Chem., vol. 275, $n^{\circ} 48$, p. 37638-37644, déc. 2000, doi: 10.1074/jbc.M006107200.

[47] S. W. Li et al., « Identification and CRISPR/Cas9 Inactivation of the C1s Protease Responsible for Proteolysis of Recombinant Proteins Produced in CHO Cells ", Biotechnol. Bioeng., vol. 0, $\mathrm{n}^{\circ}$ 0, doi: 10.1002/bit.27016.

[48] H. Eriksson et M. H. Nissen, " Proteolysis of the heavy chain of major histocompatibility complex class I antigens by complement component C1s ", Biochim. Biophys. Acta BBA - Protein Struct. Mol. Enzymol., vol. 1037, n² 2, p. 209-215, févr. 1990, doi: 10.1016/01674838(90)90169-G.

[49] H. Sakiyama et al., " Complement Cls, a classical enzyme with novel functions at the endochondral ossification center: immunohistochemical staining of activated Cls with a neoantigen-specific antibody ", Cell Tissue Res., vol. 288, n 3 , p. 557-565, mai 1997, doi: 10.1007/s004410050841.

[50] Y. Cai, B. H. D. Teo, J. G. Yeo, et J. Lu, « C1q Protein Binds to the Apoptotic Nucleolus and Causes C1 Protease Degradation of Nucleolar Proteins », J. Biol. Chem., vol. 290, n 37, p. 22570-22580, nov. 2015, doi: 10.1074/jbc.M115.670661.

[51] J. Lu et U. Kishore, « C1 Complex: An Adaptable Proteolytic Module for Complement and NonComplement Functions », Front. Immunol., vol. 8, p. 592, 2017, doi: 10.3389/fimmu.2017.00592.

[52] I. Bally et al., «Two Different Missense C1S Mutations, Associated to Periodontal Ehlers-Danlos Syndrome, Lead to Identical Molecular Outcomes », Front. Immunol., vol. 10, p. 2962, 2019, doi: 10.3389/fimmu.2019.02962. 
[53] J. G. Yeo et al., "Proteolytic inactivation of nuclear alarmin high-mobility group box 1 by complement protease C1s during apoptosis », Cell Death Discov., vol. 2, p. 16069, 2016, doi: 10.1038/cddiscovery.2016.69.

[54] B. L. Rapoport et al., " High Mobility Group Box 1 in Human Cancer », Cells, vol. 9, $n^{\circ}$ 7, juill. 2020, doi: $10.3390 /$ cells9071664.

[55] N. S. Merle, P. Singh, J. Rahman, et C. Kemper, « Integrins meet complement: The evolutionary tip of an iceberg orchestrating metabolism and immunity », Br. J. Pharmacol., juin 2020, doi: 10.1111/bph.15168.

[56] R. J. Motzer et al., " Molecular Subsets in Renal Cancer Determine Outcome to Checkpoint and Angiogenesis Blockade ", Cancer Cell, vol. 38, nº 6, p. 803-817.e4, déc. 2020, doi: 10.1016/j.ccell.2020.10.011.

[57] C. Luo, M. Chen, A. Madden, et H. Xu, « Expression of complement components and regulators by different subtypes of bone marrow-derived macrophages ", Inflammation, vol. 35, $\mathrm{n}^{\circ} \mathrm{4}, \mathrm{p}$. 1448-1461, août 2012, doi: 10.1007/s10753-012-9458-1.

[58] P. Gulati, C. Lemercier, D. Guc, D. Lappin, et K. Whaley, « Regulation of the synthesis of C1 subcomponents and C1-inhibitor ", Behring Inst. Mitt., n 93, p. 196-203, déc. 1993.

[59] P. Riihilä et al., "Tumour-cell-derived complement components $\mathrm{C} 1 \mathrm{r}$ and $\mathrm{C} 1 \mathrm{~s}$ promote growth of cutaneous squamous cell carcinoma ", Br. J. Dermatol., vol. 182, n³ 3, p. 658-670, mars 2020, doi: 10.1111/bjd.18095. 


\section{Figure legends}

Figure 1: Complement activation is associated with poor prognosis in ccRCC. A) Plasma C4d concentration determined by ELISA in cohort 2, mean+2SD represents the cut off to determine a high (i.e. above the normal range) vs. low (i.e. within the normal range) expression. B) Plasma C4d concentration $(\mathrm{ng} / \mathrm{mL})$ determined by ELISA in cohort 3, mean+2SD represents the cut off to determine a High vs. low expression. C) Progression-free survival (PFS) of ccRCC patients according to C4d plasma level with mean+2SD cut-off for cohort 2. D) PFS of ccRCC patients according to C4d plasma level with mean+2SD cut-off for cohort 3. E) Association between C4d plasma level and C4d deposits within the tumor in cohort 3, Mann-Whitney test is applied to test the difference between two groups. F) PFS of ccRCC patients of cohort 3 according to C4d deposition level within the tumor. G) IF staining of C1q (green) and C1s staining (red). H) IF staining of C4d (green) and C1s staining (red). I) PFS of ccRCC patients according to C4d plasma level and C4d deposits within the tumor in cohort 3.

Figure 2: Complement-rich phenotype of ccRCC tumors. A) Heatmap with a column scale centred represented the expression $(\log 2(1+T P M))$ of complement genes $(C 1 Q A, C 1 R, C 1 S$, $C 2, C 3$, and C4) in each ccRCC patient from the ccRCC TCGA cohort [19], [20].. Two clusters of patients could be distinguished according to the expression of all the complement genes. B) UMAP representing Seurat cluster (resolution=. 1, Dim 1:10) in 2 samples of one ccRCC patient, analysed by scRNA-seq [22]. C) UMAP plot representing the expression of C1QA, $C 1 R, C 1 S, C 2$ and $C 3$ in cells of the ccRCC patient, analysed by scRNA-seq and zoom in the tumor cell clusters. In this analysis, $C 4 A$ and $C 4 B$ were not included because very low number of cells expressed these genes in the tested patient. D) Expression level of $C 1 R, C 2$, and $C 3$ in tumor cells positive for C1S or negative for C1S, based on the scRNA-seq data. If C1s counts $>0$, the cell is considered as positive. E) IF staining of C1s (red) and C4 (green). F) IF staining of C1s (red) and C3 (green). G) Consecutive sections of a ccRCC tumor stained by $\mathrm{IHC}$ for $\mathrm{C} 1 \mathrm{~s}, \mathrm{C} 4$, and C3.

Figure 3. C1s is associated with poor prognosis in ccRCC. A) C1S expression (log2TPM) in cCRCC tumors versus normal kidney from TCGA normal and GTEx database (GTEx Analysis Release V8 (dbGaP Accession phs000424.v8.p2). B) C1S expression (log2TPM) in different TNM stages of ccRCC tumors generated by GEPIA. C) Kaplan-Meier curves of overall survival (OS) according to C1S gene expression level using median cut-off on ccRCC TCGA cohort, 
using Protein Atlas. D-E) Kaplan-Meier curves of D) PFS and E) OS according to the C1s classification in three groups in cohort 1 . Group 0 : very low $\%$ of cells $(<5 \%)$, group 1 : intermediate $\%$ of cells (5-30\%), group 2: high \% of cells (>30\%). F) Kaplan-Meier curves of PFS according to the C1s classification in three groups in cohort 3. G) Correlation between Stage and $\mathrm{C} 1 \mathrm{~s}$ in cohort 1. H) Correlation between Fuhrman grade and C1s in cohort 1. I) Correlation between Metastatic status (0: negative; 1: positive) and C1s in cohort 1. J) Kaplan-Meier curves according to C1s gene expression level using optimal cut-off on J) Stage I patients from the KIRC TCGA cohort, visualized using Protein Atlas tool. K-L) Kaplan-Meier curves of PFS according to C1s protein staining on stage I patients from K) cohort 1 and $\mathrm{L}$ ) cohort 3. For the Kaplan-Meier curves log-rank test was used and $p<0.05$ was considered significant.

Figure 4: C3aR and C5aR1 may impact survival in cCRCC by action on macrophage infiltration or tumor cells. A) C3AR and C5AR1 gene expression impacts survival in the cCRCC TCGA cohort, at optimal cut-off. B) sCRNA-seq shows that macrophages and neutrophils as well as some rare tumor cells express C3AR and C5AR1. C) IF staining of CCRCC tumors (CD163 in green, C5aR1 in red) D) Density of CD163 ${ }^{+}$cells in each group of C1s classification determined by IHC in cohort 1. E) Kaplan-Meier curve of PFS according to the combination of C1s and CD163 High/Low classification in cohort 1. F) IHC for C5aR1 on human tonsil (a positive control for immune cell staining) and ccRCC tumors (shown are two representative ccRCC tumors from $n=11$ ). Immune infiltrating cells were detected in every tumor but C5aR1 positive tumor cells were found only in 1/11 tumors. G) Caki-1 cells express low levels of C3aR and C5aR1, detected by flow cytometry. H-J) Incubation of Caki-1 with purified C3a or $\mathrm{C} 5 \mathrm{a}$ does not impact $\mathbf{H}$ ) viability of the cells, detected by flow cytometry, error bars represent mean with SD; I) morphology, detected by actin staining, revealed by immunocytofluorescence; or J) proliferation, detected by flow cytometry, error bars represent mean with SD. K-L) C3a but not C5a impacts the migration of Caki-1 cells, evaluated by a scratch test, error bars represent mean with SD.

Figure 5. Modulation of tumor cell gene expression by C1s. A) Heatmap representing the most variable genes between $\mathrm{C}^{+} \mathrm{S}^{+}$tumor cells and $\mathrm{C}^{-} \mathrm{S}^{-}$tumor cells. Differential genes are determined using FindAllmarker function from Seurat package in R. Genes with a log 2 fold change $>0.5$, min.pct $=0.25$, min.diff.pct $=0.25$ and an adjusted $p$-value $<0.05$ are shown. Red colors represent high expression and blue colour low expression. The genes are ordered 
according to the adjusted $\mathrm{p}$ value. B) Gene ontology (GO) analysis of functional annotations for up- or downregulated genes in $\mathrm{C}^{+} \mathrm{S}^{+}$tumor cells in comparison with $\mathrm{C} 1 \mathrm{~S}^{-}$tumor cells. All genes with min.pct $=0.25$ and adjusted $p$ value $<0.05$ are included in the analysis $\mathbf{C}$ ) Reactome analysis of up- or downregulated genes in $\mathrm{C}_{1 S^{+}}$tumor cells in comparison with $\mathrm{C}^{-} \mathrm{S}^{-}$tumor cells. D) Top 50 differentially expressed genes after silencing with siC1s compared with siC in Caki-1 cells. The most differentially expressed genes (log2 counts) were selected on adjusted p-value. Expression across each gene has been row scaled with relatively high expression of a given gene marked in red and samples with relatively low expression marked in blue. Lighter shades and white represent genes with intermediate expression levels. E) Volcanoplot depicting the results of differential expression obtained with DESeq2 analysis between Caki-1 siC1s $(n=3)$ and Caki-1 siC $(n=3)$. The volcano plot was generated with $\mathrm{R}$ software and EnhancedVolcano package. Red points represent genes with a log 2 fold change $>1$ and $p$-value adjusted $<0.05$. F) GO analysis of functional annotations for up- or downregulated genes by C1s silencing vs. Caki-1 siC. G) Reactome analysis of upor downregulated genes by $\mathrm{C} 1 \mathrm{~s}$ silencing vs. Caki-1 siC.

Figure 6. C1s expression modulates proliferation and viability of Caki-1 cells. A) Viability assessment by the percentage of DAPI-positive dead cells, determined by flow cytometry after Caki-1 siC1s or Caki-1 siC were cultured for 72 hours. Data shown are from 11 independent experiments, error bars represent mean with SD. B) Histogram representing CFSE intensity in Caki-1 siC1s and Caki-1 siC after $72 \mathrm{~h}$ of proliferation (green = unstained Caki-1 cells, orange $=$ Caki- 1 , taken at the time of CFSE staining T0, red $=$ Caki- 1 siC after $72 \mathrm{~h}$ of proliferation, blue $=$ Caki-1 siC1s after $72 \mathrm{~h}$ of proliferation). C) Fold change of CFSE mean fluorescence intensity (MFI) in Caki-1 siC1s vs. Caki-1 siC on 4 independent experiments. D) IF staining of phalloidin (white) in Caki-1 siC1s and Caki siC. E) Fold change of CFSE mean fluorescence (MFI) in Caki-1 siC vs siC1s vs siC1s with addition of purified protein C1s enzyme or proenzyme.

Figure 7. C1s production by tumor cells impacts CD8 T cells. A) C1s secretion in the supernatant after treatment of Caki-1 cells with IFNY (0, 100 and $1000 \mathrm{ng} / \mathrm{mL}$ ), detected by ELISA, error bars represent mean with SD. B-C) Expression of MHC class I A/ B/C, as assessed by by flow cytometry, on B) resting and C) IFNp-activated Caki-1 cells, untreated, siC or siC1s. D) Kaplan-Meier curve of PFS, with patients in cohort 1 stratified by $\mathrm{CD} 8^{+}$T-cell density. E) Correlation between the expression of C1S (Log2TPM), separated in quartiles and CD8 
(Log2TPM) from TCGA cohort, the T-cell signature was identified by the MCP-counter tool [21]. F) Density of $C D 8^{+}$cells in each group of C1s classification determined by IHC, Cohort 1. G) Density of PD- $1^{+}$cells in each group of C1s classification determined by IHC, Cohort $1 . \mathbf{H}$ M) Co-culture of activated sorted $\mathrm{CD}^{+}(\mathbf{H}-\mathrm{J})$ and $\mathrm{CD}^{+}$(K-M) T cells with untreated, siC or siC1s Caki 1 cells, error bars represent mean with SD. $\mathbf{H}, \mathbf{K}) \%$ of $\mathrm{CD}_{3} 8^{+}$cells among $\mathrm{CD}^{+}(\mathbf{H})$ and $\mathrm{CD}^{+}$(K) T cells cultured for $24 \mathrm{~h}$ alone or in the presence of siC or siC1s Caki-1 cells at a ratio of 5 tumor cells to $1 \mathrm{~T}$ cell $(5: 1)$. I,L) \% of $\mathrm{CD} 8^{+} \mathrm{T}$ cells after $24 \mathrm{~h}$ of co-culture, with varying tumor cell to $\mathrm{T}$ cell ratios. J-M) \% of $\mathrm{CD} 38^{+} \mathrm{T}$ cells (CD4 (J) or $\mathrm{CD} 8^{+} \mathrm{T}$ cells (M)) after $24 \mathrm{~h}$ of co-culture, with addition of purified C1s enzyme + proenzyme in the supernatant of the co-culture of the siC1s (siC1s+C1s condition) cells. Albumin (Alb) was used as an irrelevant protein control. N) Proposed mechanism linking the complement-rich phenotype of the ccRCC cells to cancer progression: At least 3 non-exclusive processes are suggested: 1) Activation of the canonical complement cascade by tumor cell-derived C1r, C1s, C3, C4 and C5 and macrophage-produced C1q. C3a could reduce tumor cell migration, whereas C5a attracts and activate macrophages. C4d deposits at the tumor-cell surface and plasma C4d, as well as macrophage infiltration and C5aR1 expression, confer poor prognosis. 2) C1s acts in a non-canonical manner inside the tumor cell, modifying its transcription program and phenotype. It promotes proliferation and survival. 3) C1s-producing tumor cells have a complex interplay with infiltrating $T$ cells. T cell-derived IFN $\gamma$ stimulates the production of C1s. The C1s-expressing tumor cells fail to activate $\mathrm{CD} 4^{+}$and $\mathrm{CD} 8^{+} \mathrm{T}$ cells. In contrast, $\mathrm{C} 1 \mathrm{~s}$ depletion in tumor cells results in aberrant expression of MHC class I and alleviates the inability of tumor cells to activate $\mathrm{CD}^{+} \mathrm{T}$ cells. The figure was generated with BioRender.com. 
A.

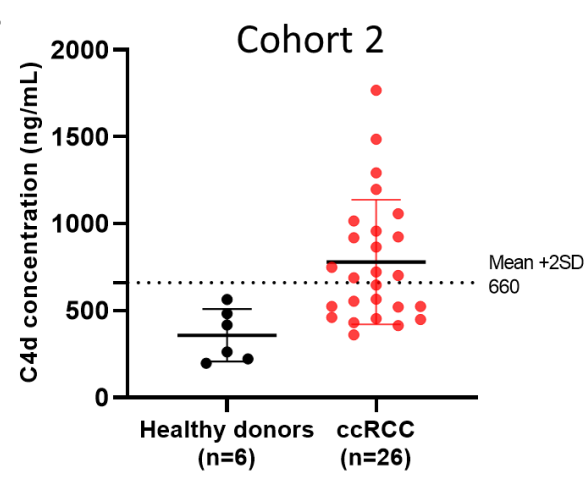

C.

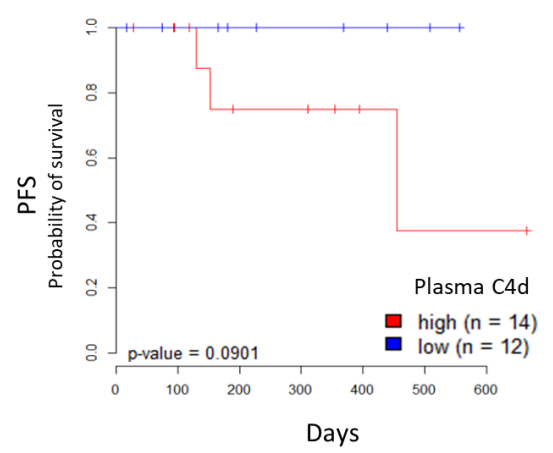

E.

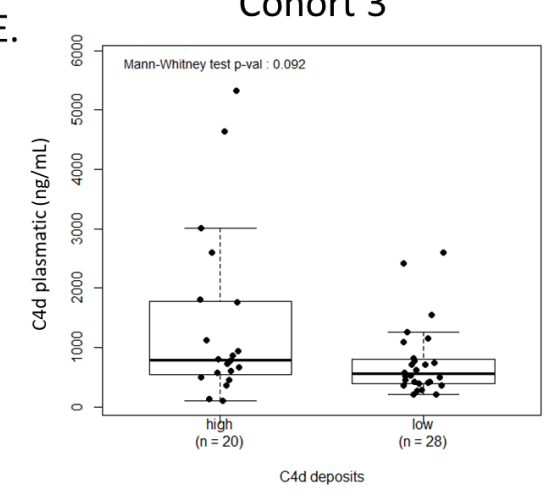

G.

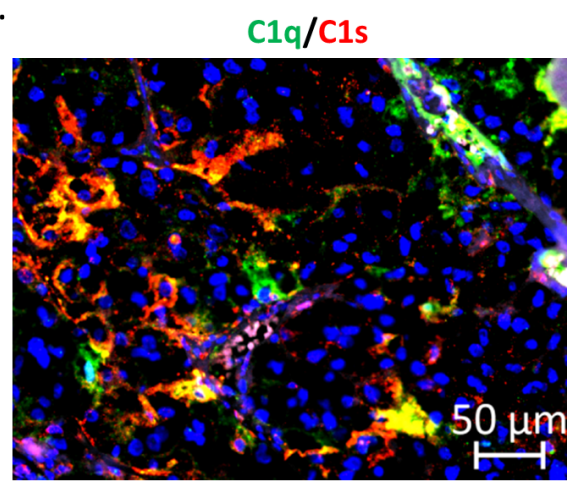

B.

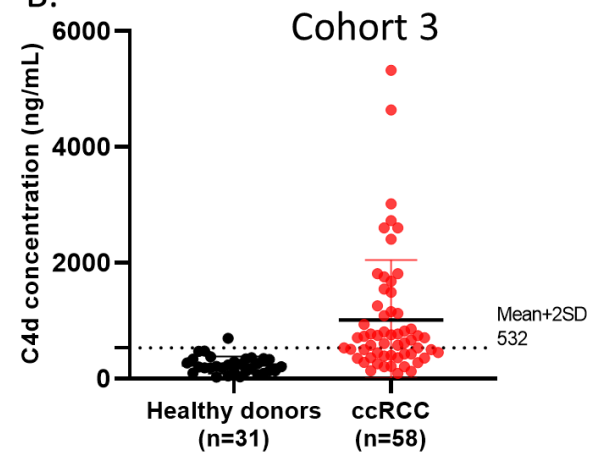

D.

Cohort 3
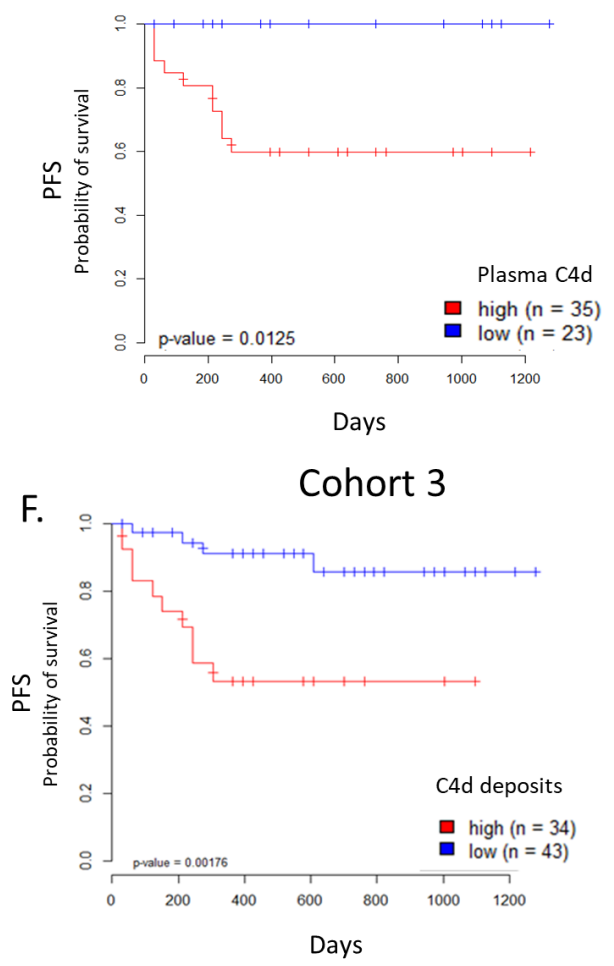

H.

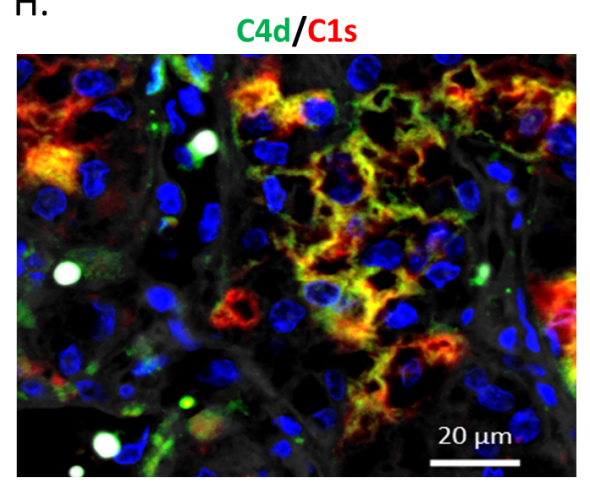

I.

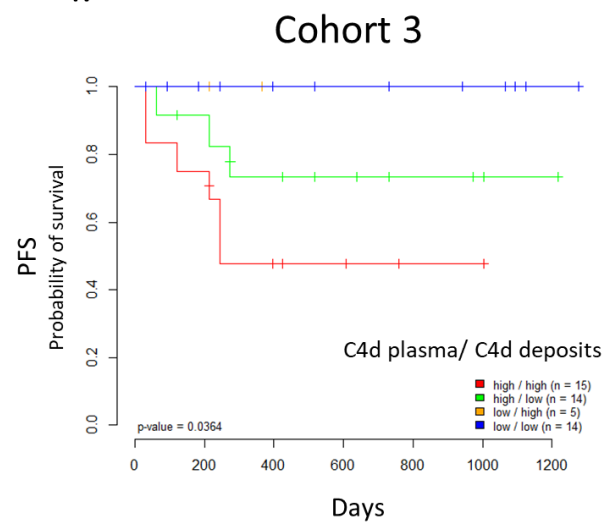

Figure 1. Complement activation, detected by intratumoral and plasma $\mathrm{C} 4 \mathrm{~d}$ is associated with poor prognosis in CCRCC 


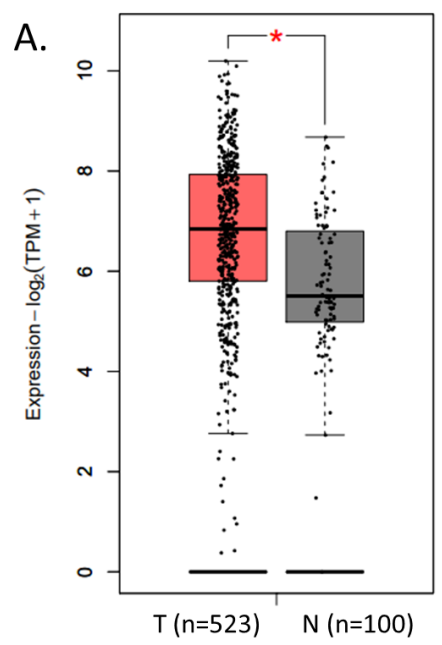

B.

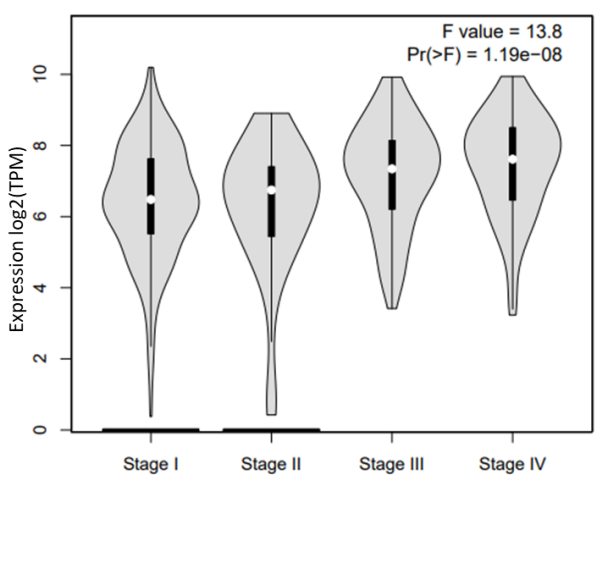

C

KIRC, Protein Atlas

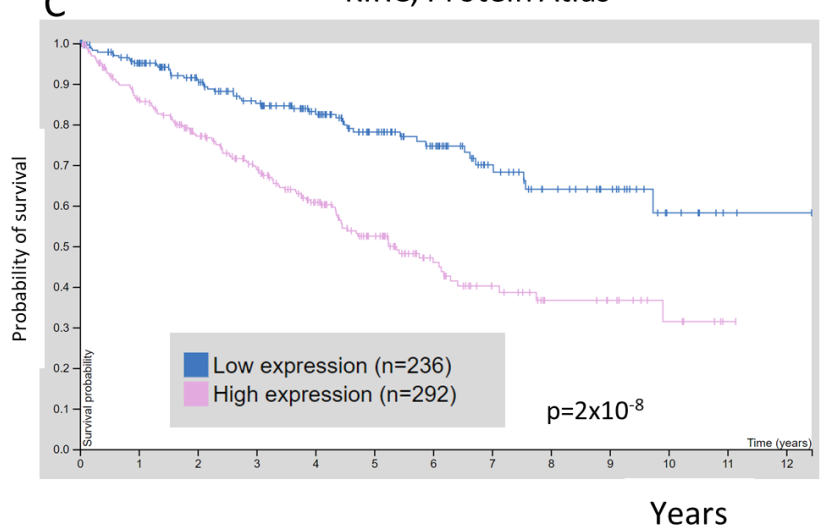

D.

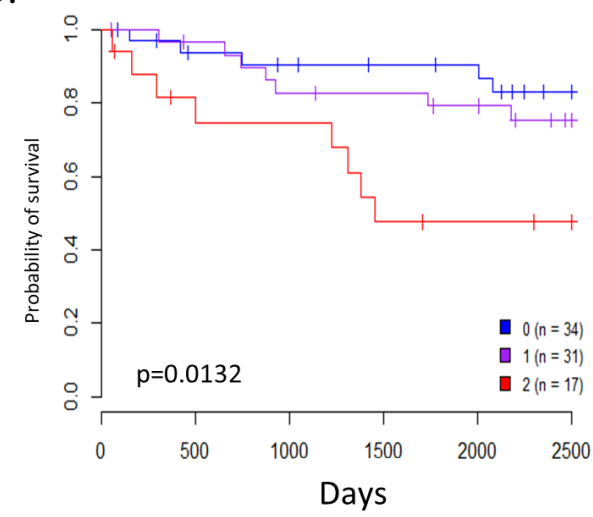

G.

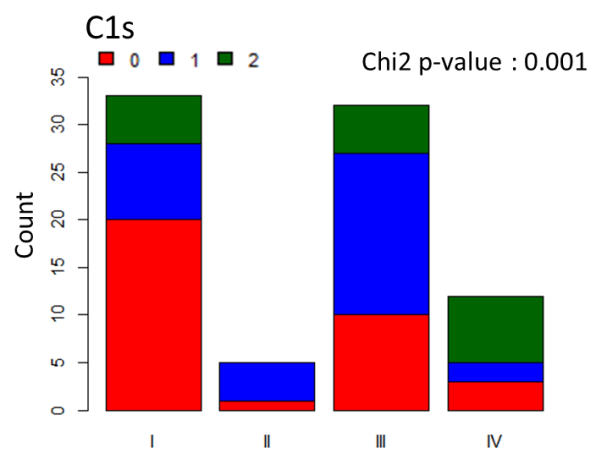

Tumor stage

J.

Stage I, KIRC, Protein Atlas

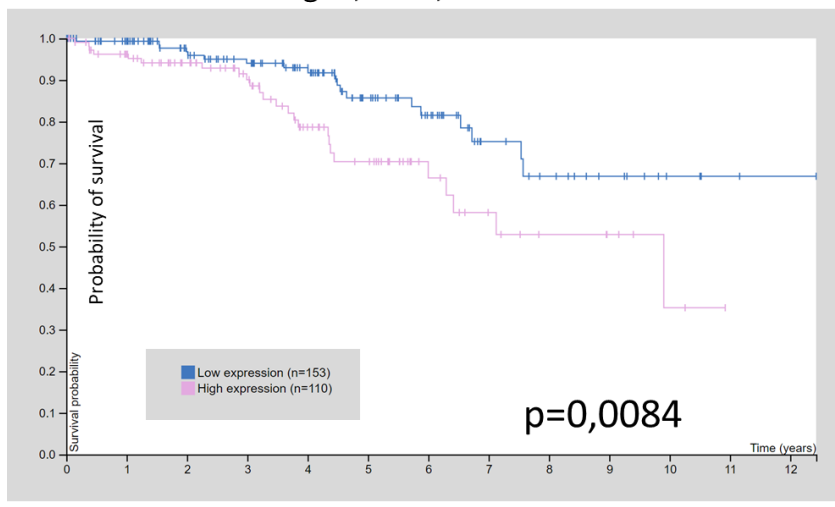

Years

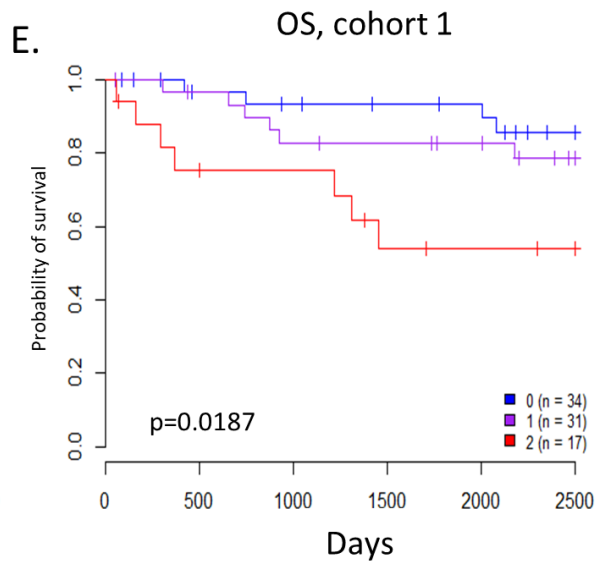

H.

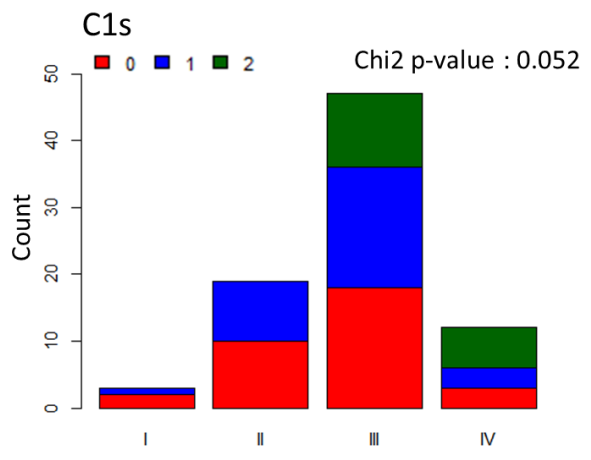

Fuhrman grade

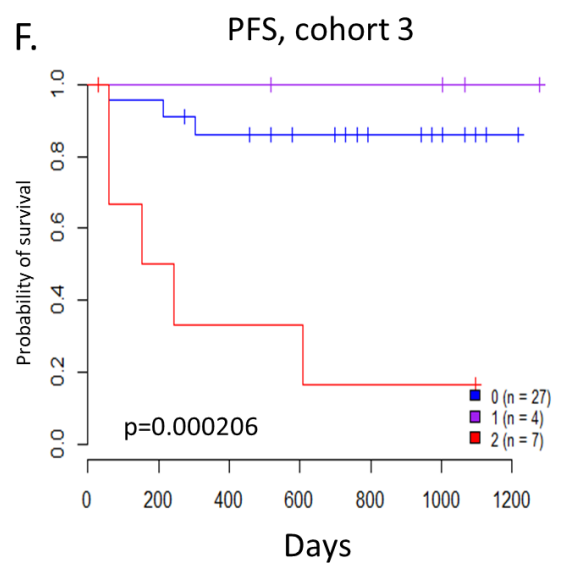

I.

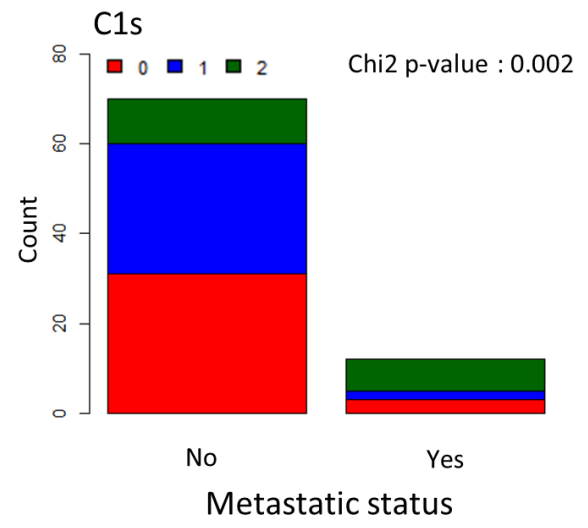

L.

PFS, stage I, cohort 1

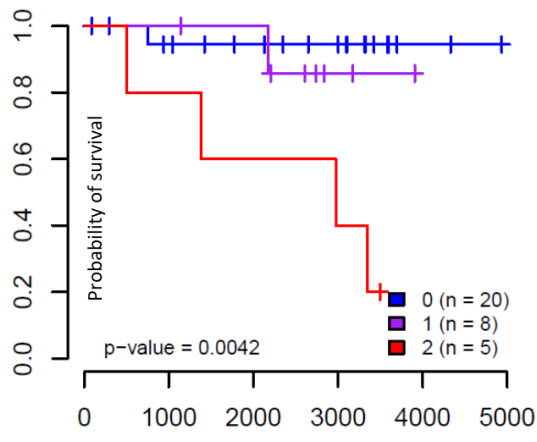

Days
PFS, stage I, cohort 3

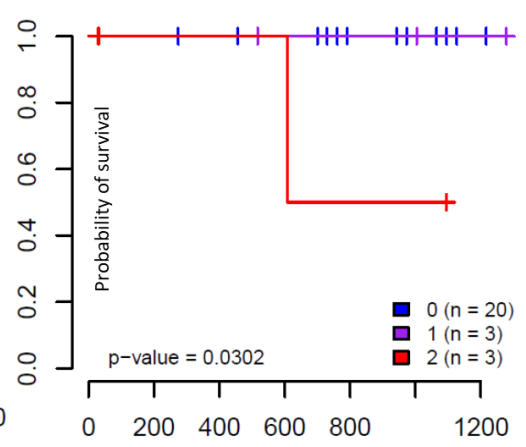

Days 


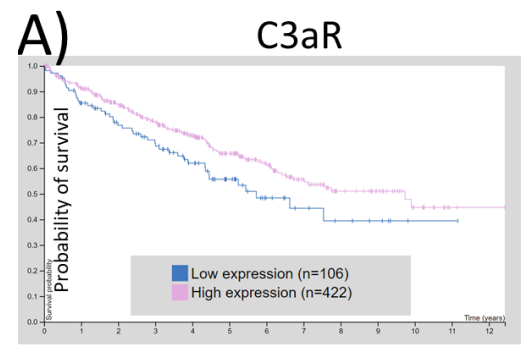

Years
C5aR1

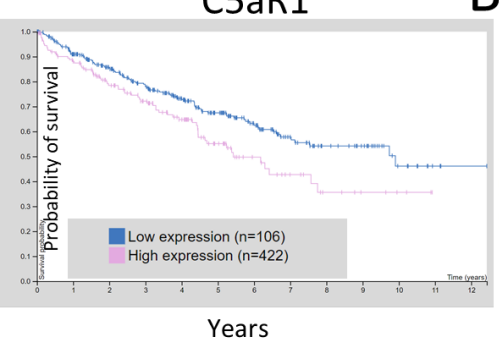

D)

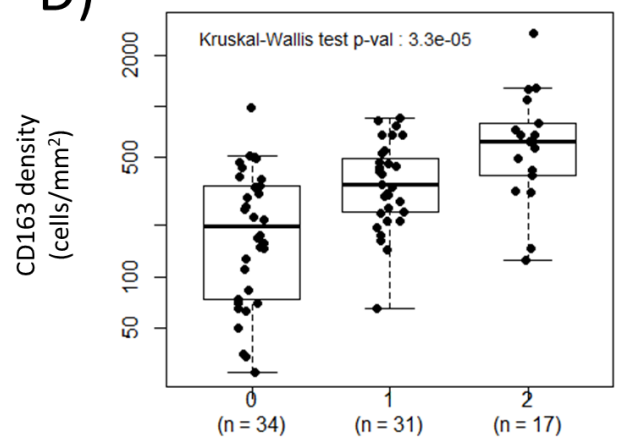

C1s
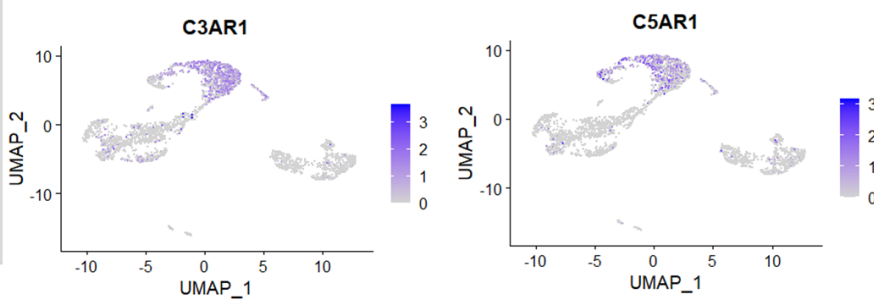

E)

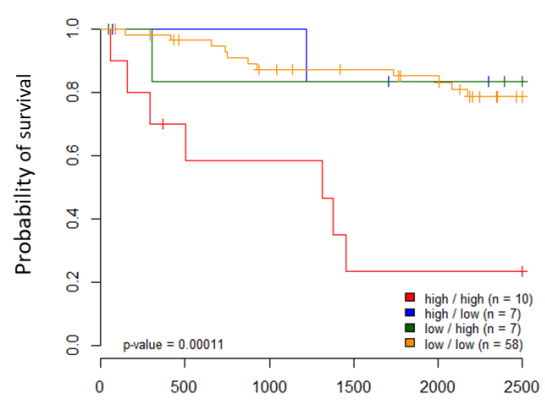

Days
F) C5aR1 +ve control

G)

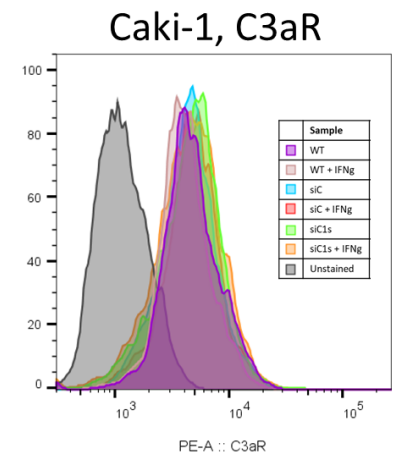

C5aR1 +ve immune cells, frequent

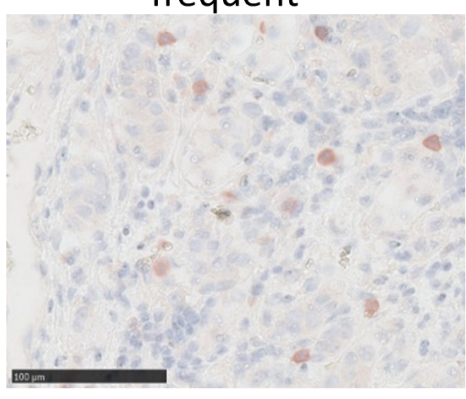

Caki-1, C5aR1

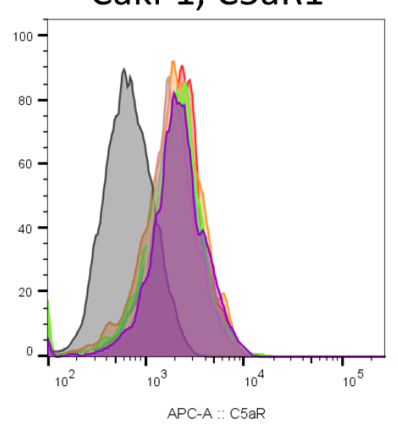

H)

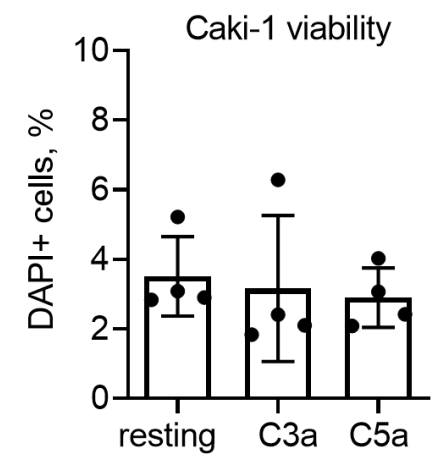

very rare

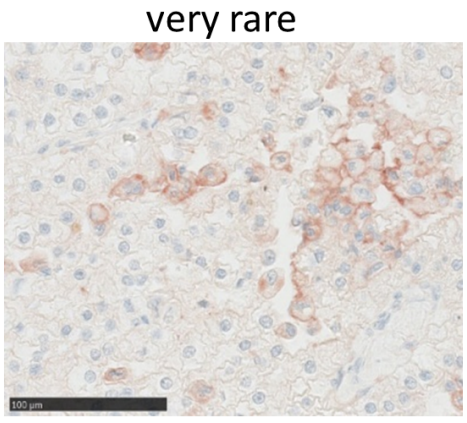

C3a

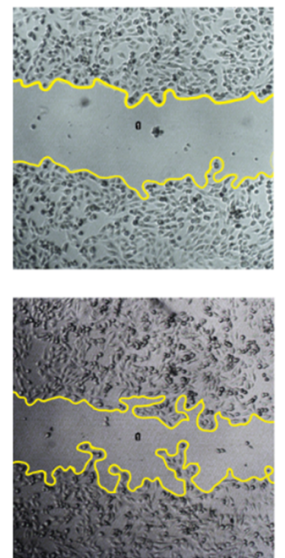

$\mathrm{C} 5 \mathrm{a}$

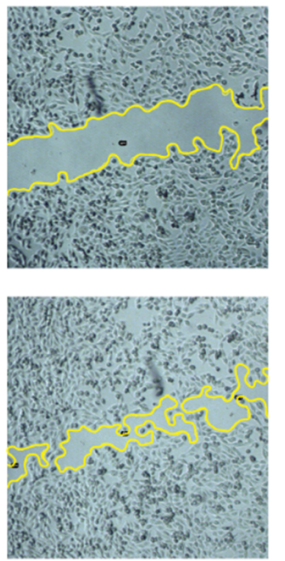

I) Caki-1, morphology
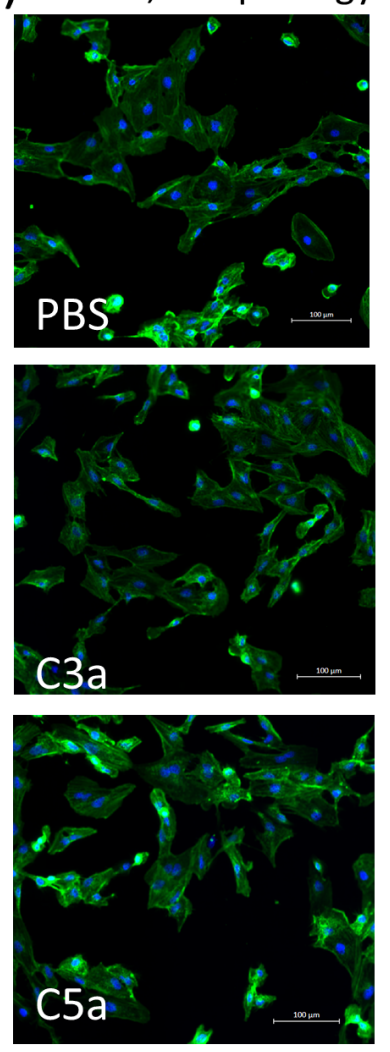

L)

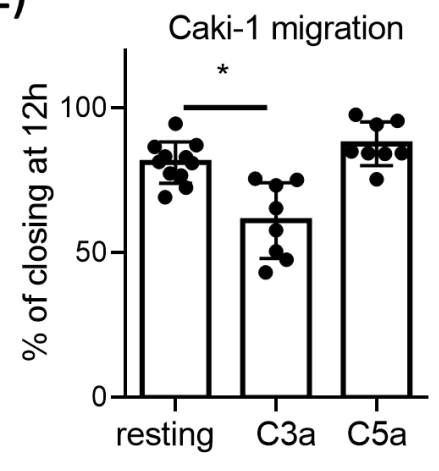

Figure 4: C3aR and C5aR1 impact survival in ccRCC likely by action on tumour cells or macrophage Down paded from cancerimmunolres.aacrjournals.org on May 27, 2021. ( 2021 American Association for Cancer Research. 
A.

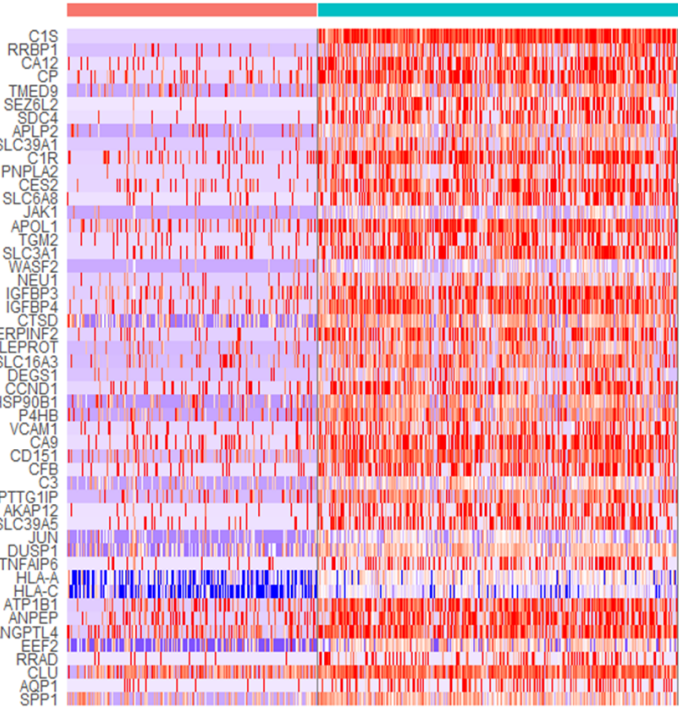

D.
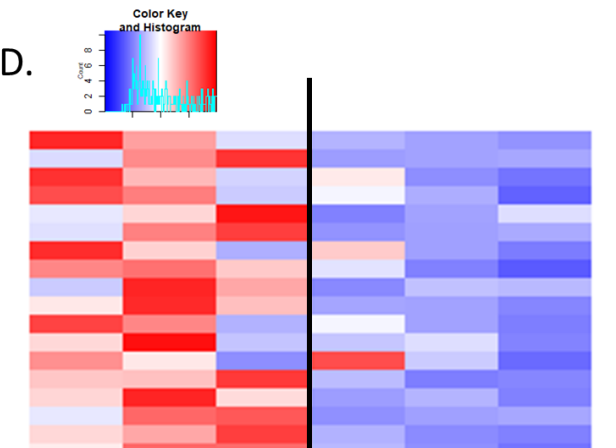

F.

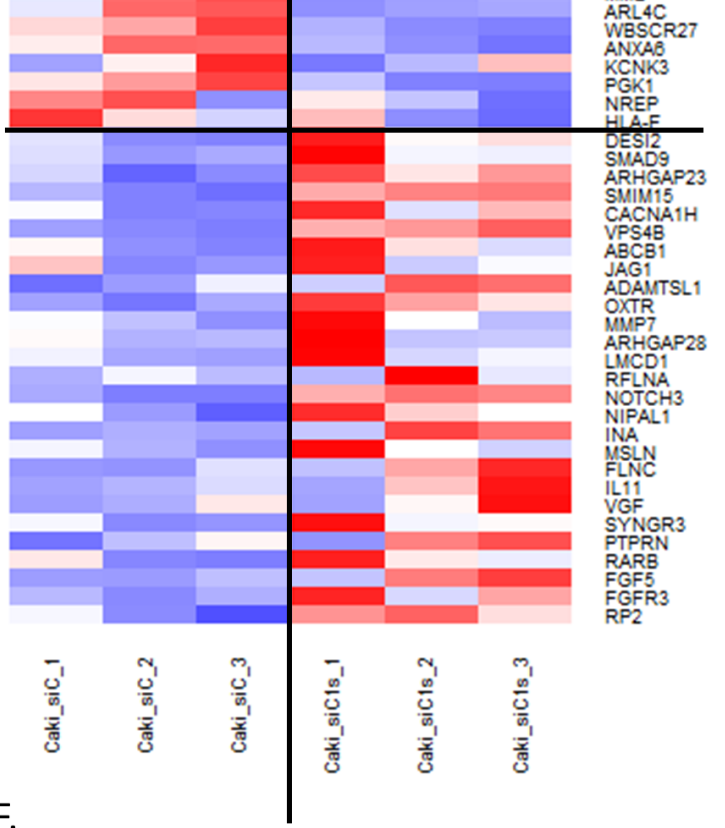

GO_Biological Process

\section{Upregulated genes}

Regulation of cell development (GO:0060284)

Negative regulation of growth (GO:004926)

Regulation of chemotaxis (GO:0050920)

Multicellular organismal signaling (GO:0035637)

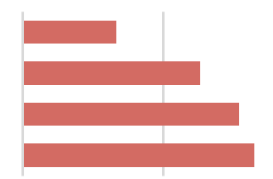

$\begin{array}{lll}0 & 5 & 10\end{array}$

Downregulated genes

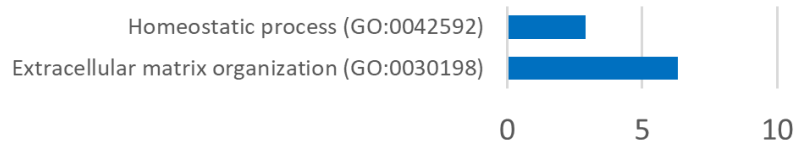

B.

GO_Biological Process

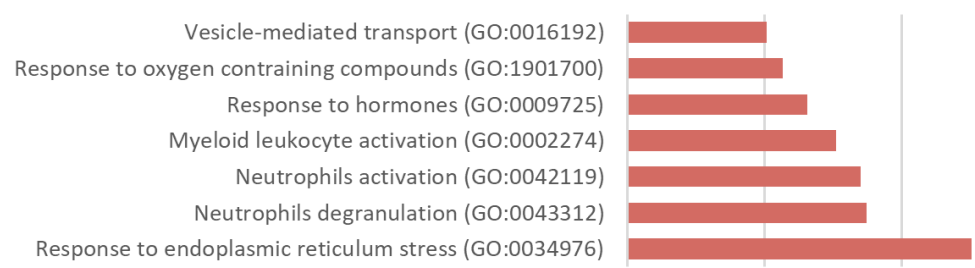

2

4

C.

\section{REACTOME}

Extracellular matrix organization (R-HSA-1474244)

Neutrophils degranulation (R-HSA-6798695)

Complement cascade (R-HAS-166658)

Regulation of IGF transport and uptake (R-HAS-381426)

Folding, assembly and peptide loading of class I MHC (R-HAS-983170)

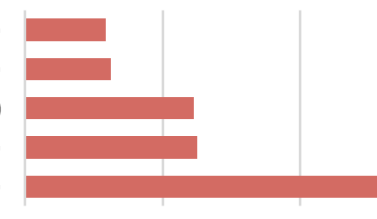

E.

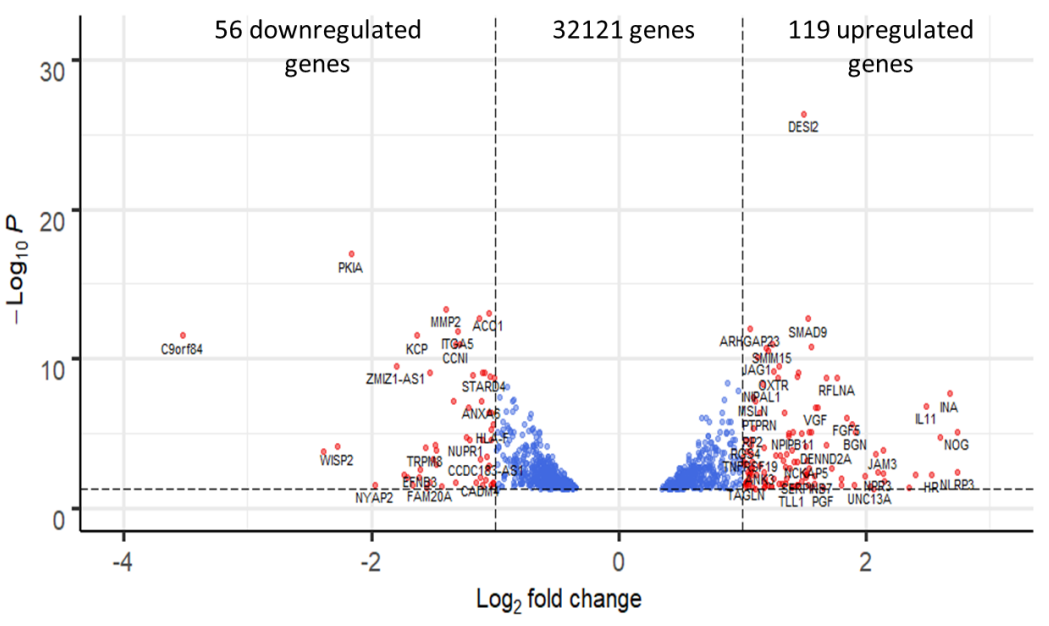

G.

\section{REACTOME}

\section{Upregulated genes}

Extracellular matrix organization (R-HSA-1474244) L1CAM interactions (R-HAS-373760 Signaling by NOTCH3 (R-HSA-9012852) Signaling by activated points mutants of FGFR3 (R-HAS-1839130)

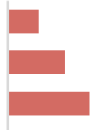$$
0
$$$$
20
$$

\section{Downregulated genes}

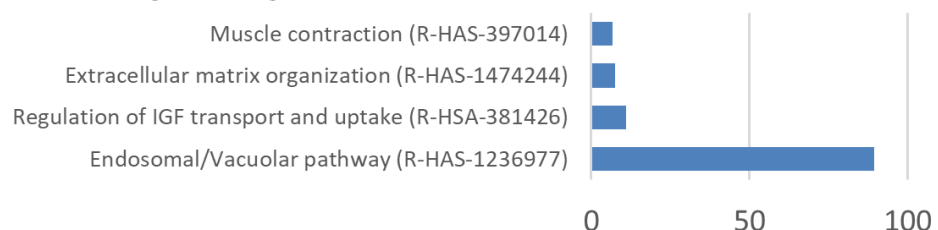

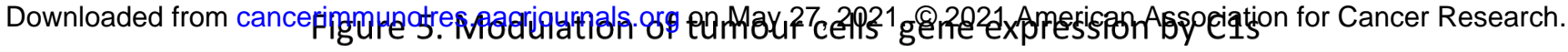



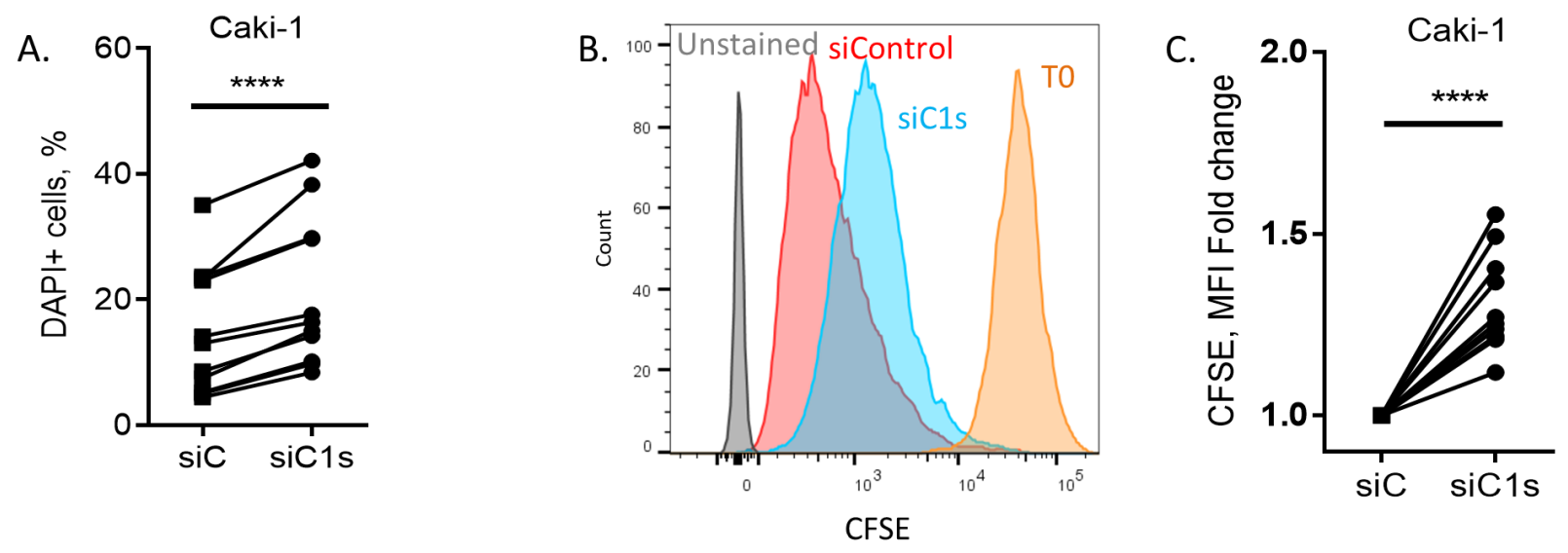

D.
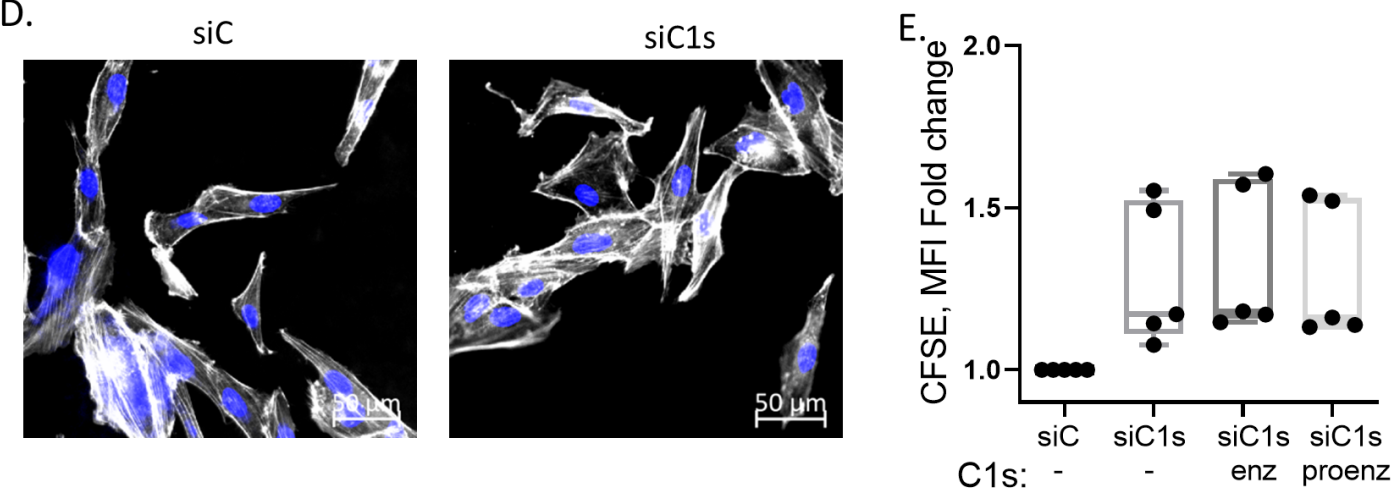

Figure 6 . C1s expression modulates proliferation and viability of Caki-1 cells 


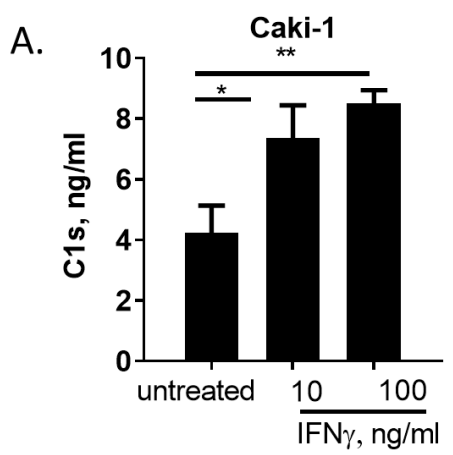

B.

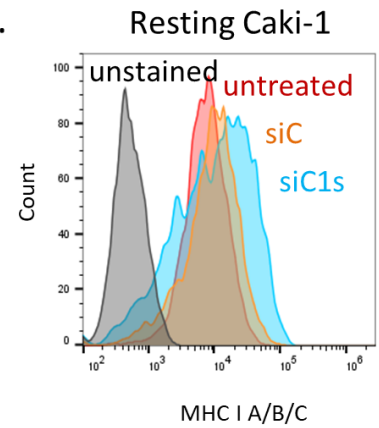

C. IFN $\gamma$ activated Caki-1

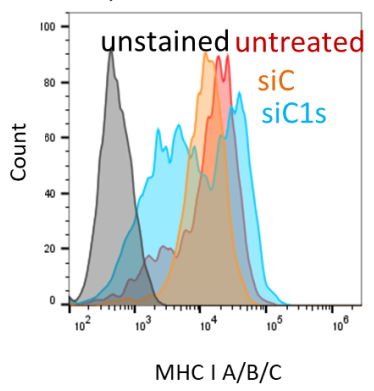

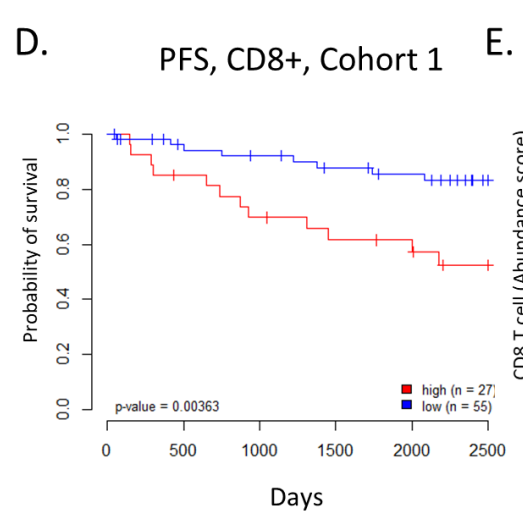

H.

activated $C D 4+T$ cells

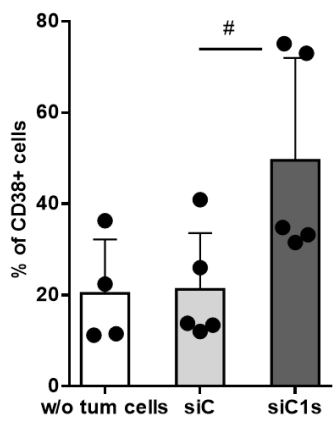

K. activated CD8+ T cells

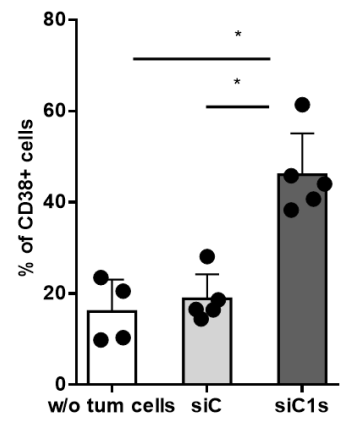

M.

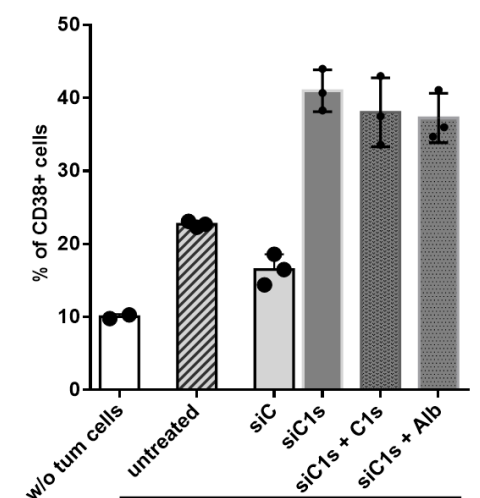

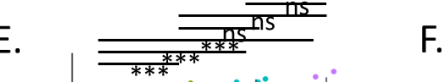
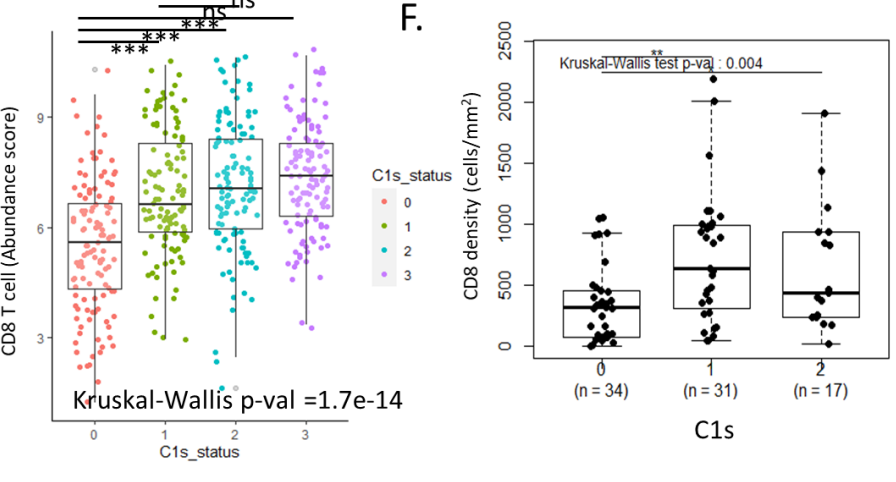

J.
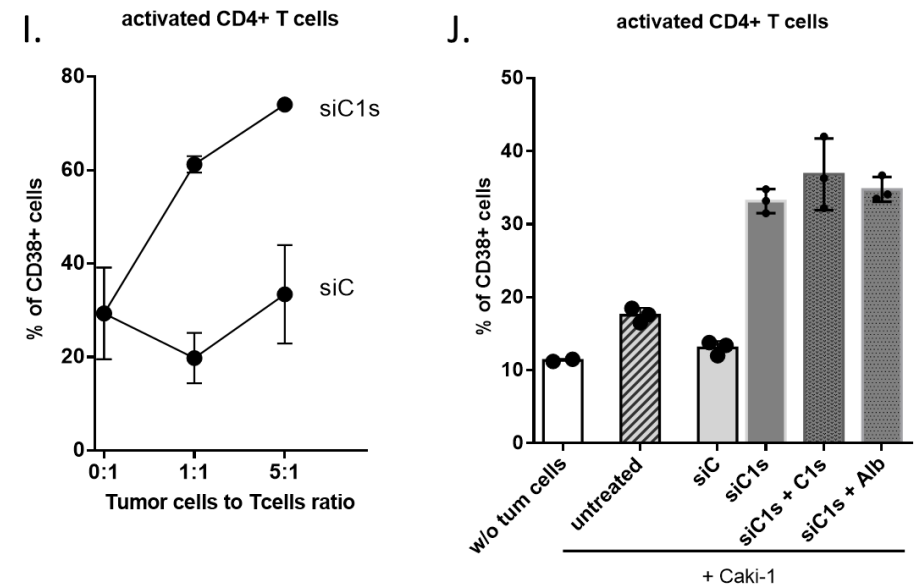

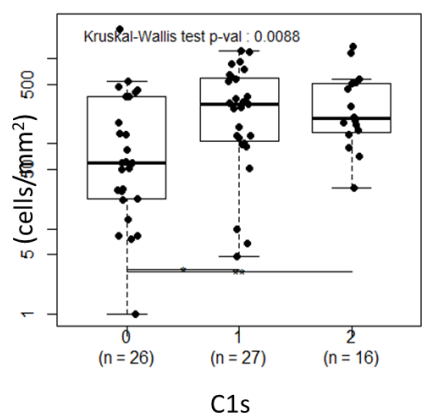

C1s

L. activated $\mathrm{CD} 8+\mathrm{T}$ cells

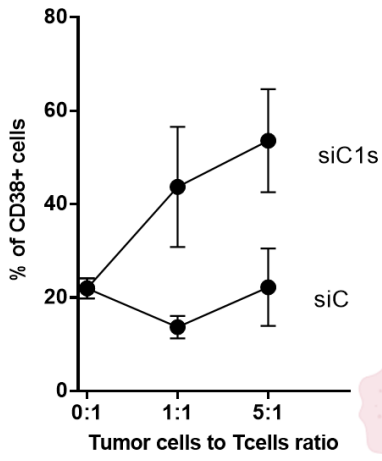

N.

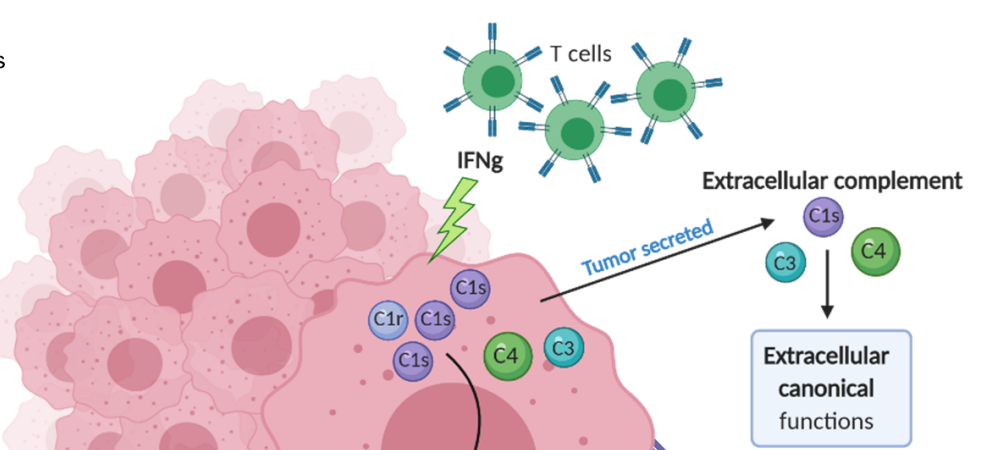

Tumor cells

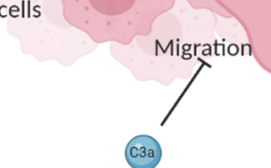

Intracellular non-canonical functions

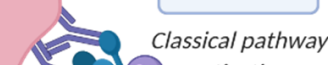

Classical pathwa
C1s activation

(1r)

(C4)
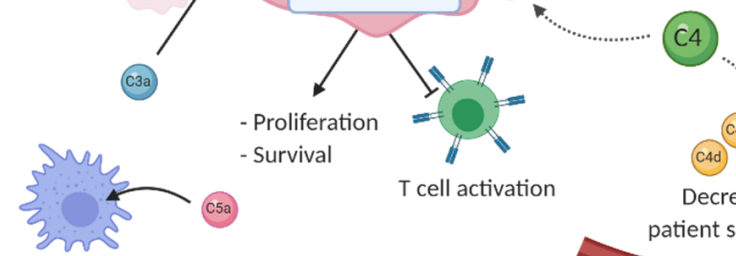

- Proliferation - Survival

T cell activation

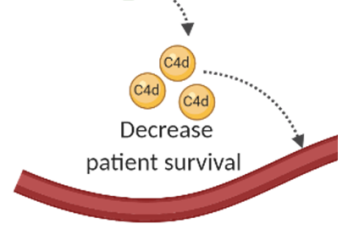




\section{Cancer Immunology Research}

\section{Complement $\mathrm{C} 1 \mathrm{~s}$ and $\mathrm{C} 4 \mathrm{~d}$ as prognostic biomarkers in renal cancer: emergence of non-canonical functions of $\mathrm{C} 1 \mathrm{~s}$}

Marie V Daugan, Margot Revel, Jules Russick, et al.

Cancer Immunol Res Published OnlineFirst May 26, 2021.

\begin{tabular}{|rl|}
\hline Updated version & $\begin{array}{l}\text { Access the most recent version of this article at: } \\
\text { doi:10.1158/2326-6066.CIR-20-0532 }\end{array}$ \\
$\begin{aligned} \text { Supplementary } \\
\text { Material }\end{aligned}$ & $\begin{array}{l}\text { Access the most recent supplemental material at: } \\
\text { http://cancerimmunolres.aacrjournals.org/content/suppl/2021/05/13/2326-6066.CIR-20-0532.D }\end{array}$ \\
$\begin{aligned} \text { Author } \\
\text { Manuscript }\end{aligned}$ & $\begin{array}{l}\text { Author manuscripts have been peer reviewed and accepted for publication but have not yet } \\
\text { been edited. }\end{array}$ \\
\hline
\end{tabular}

E-mail alerts Sign up to receive free email-alerts related to this article or journal.

Reprints and To order reprints of this article or to subscribe to the journal, contact the AACR Publications Subscriptions Department at pubs@aacr.org.

Permissions To request permission to re-use all or part of this article, use this link http://cancerimmunolres.aacrjournals.org/content/early/2021/05/25/2326-6066.CIR-20-0532. Click on "Request Permissions" which will take you to the Copyright Clearance Center's (CCC) Rightslink site. 